\title{
Cylindrical shells under uniform bending in the framework of Reference Resistance Design
}

\author{
Jie Wang ${ }^{1}$, O. Kunle Fajuyitan ${ }^{2}$, M. Anwar Orabi ${ }^{3}$, \\ J. Michael Rotter ${ }^{4} \&$ Adam J. Sadowski ${ }^{5}$
}

\begin{abstract}
The resistance of cylindrical shells and tubes under uniform bending has received significant research attention in recent times, with a number of major projects aiming to characterise their strength through both experimental and numerical studies. However, the investigated cross-section slenderness ranges have mostly addressed low radius to thickness ratios where buckling occurs after significant plasticity and the influence of geometric imperfections is relatively minor. The behaviour under uniform bending of thinner imperfection-sensitive cylinders that fail by elastic buckling was largely omitted, as was the influence of finite length effects. The value of such resistance models that are only useful for thicker cylinders is therefore somewhat limited.

This paper offers the most comprehensive known characterisation of the buckling and collapse resistance of isotropic cylindrical shells and tubes under uniform bending. Expressed within the modern framework of Reference Resistance Design (RRD), it holistically incorporates the effects of material plasticity, geometric nonlinearity and sensitivity to realistic and damaging weld depression imperfections. The characterisation was made possible by the authors' recently-developed novel methodology for mass automation of nonlinear shell buckling finite element analyses. A modification of the RRD formulation is proposed which facilitates its application to systems of low slenderness, and offers a compact algebraic characterisation of all potential imperfection amplitudes for this common shell structural condition. A reliability analysis is also performed.
\end{abstract}

\section{Keywords}

Cylindrical shells; uniform bending; Reference Resistance Design; plasticity; geometric nonlinearity; imperfection sensitivity.

\footnotetext{
${ }^{1}$ Lecturer, Department of Architecture and Civil Engineering, University of Bath

${ }^{2} \mathrm{PhD}$ Graduate, Department of Civil and Environmental Engineering, Imperial College London

${ }^{3} \mathrm{PhD}$ Candidate, Department of Building Services Engineering, The Hong Kong Polytechnic University

${ }^{4}$ Visiting Professor, Department of Civil and Environmental Engineering, Imperial College London

${ }^{5}$ Senior Lecturer, Department of Civil and Environmental Engineering, Imperial College London
} 


\section{Introduction}

Uniform bending is one of the most common reference loading arrangements for cylindrical metal shells, manifest in chimneys, wind turbine towers, piles, pipelines, structural members (circular hollow sections - CHS), aerospace vehicle fuselages, silos and tanks. These applications and others involve a wide range of radius to thickness $(r / t)$ ratios, characteristic lengths and manufacturing or fabrication processes. The thickest cylinders fail by plastic collapse whilst the thinnest fail by elastic buckling, with those inbetween involving varying degrees of plasticity at failure. In addition, long cylinders under bending have long been known to exhibit ovalisation of the cross-section [1], a geometrically nonlinear phenomenon that greatly degrades the elastic load-bearing resistance. Construction or manufacturing defects may further degrade the moment capacity, so that a truly comprehensive design resistance description must be able to represent failure mechanisms at all lengths and cross-section slendernesses by combining the effects of geometric nonlinearity, plasticity and imperfection sensitivity, with each one having different impacts on different geometries.

The testing of 'thick' cylinders (tubes) under bending has a long history, with many research programmes exploring their bending capacities e.g. [2-8]. However, the resulting data sets were typically used to calibrate resistance functions constructed at the level of abstraction of classical beam theory, an understandable choice for investigations that aimed to establish cross-section slenderness limits in relation to failure through full plasticity or first yield. However, more slender cylinders must be treated as shells: their strength is governed by local buckling, ovalisation and imperfection sensitivity, effects that are difficult to reconcile with a beam theory treatment without conservative and empirical adjustments that are based on a relatively weak test database. The review report of Rotter and Sadowski [9] explored the hand calculation predictions of a selection of international standards for the bending resistance of tubes and cylinders of intermediate slenderness and showed that the literature includes a great range of such empirical predictions that give quite different results. This indicates that no reconciliation currently exists between 'beam' and 'shell' treatments. This paper aims to establish such a reconciliation, based on the framework of Reference Resistance Design [10-12] for the manual dimensioning of metal shell systems. 


\section{Reference Resistance Design (RRD)}

The Reference Resistance Design (RRD) framework was recently devised by Rotter [1012]. It permits any structural system to be algebraically characterised in a consistent and conservative manner, relating the characterisation directly to the mechanical phenomena. This allows a designer to have rapid access to the outcome of fully nonlinear resistance assessments of a structural system without recourse to onerous computational analyses. For cylinders under uniform bending, $R_{k}(\lambda) \equiv M_{k}(\lambda)$ is the characteristic value of the nonlinear buckling moment (based on the characteristic values of material properties), while the two reference resistances $R_{p l} \equiv M_{p l}$ and $R_{c r} \equiv M_{c r}$ are the full plastic and linear elastic critical buckling moments respectively. Ignoring length effects, these resistances may be obtained from small displacement theory as:

$$
M_{p l}=\left(4 r^{2} t+\frac{1}{3} t^{3}\right) \sigma_{y} \approx 4 r^{2} t \sigma_{y} \text { as } \frac{r}{t} \rightarrow \infty \text { and } M_{c r}=\frac{\pi}{\sqrt{3\left(1-v^{2}\right)}} \operatorname{Ert}^{2}
$$

where $\sigma_{y}, E$ and $v$ are the yield stress, elastic modulus and Poisson ratio respectively, while $r$ and $t$ are the cylinder middle surface radius and thickness respectively.

$\mathrm{RRD}$ is built on a base relationship between a system's characteristic failure resistance $\chi$ and its dimensionless slenderness $\lambda$, which can be illustrated by both the 'traditional' and Rotter's [13,14] 'modified' forms in Fig. 1. The most complete current functional form characterising this 'capacity curve' is as follows [15-17]:

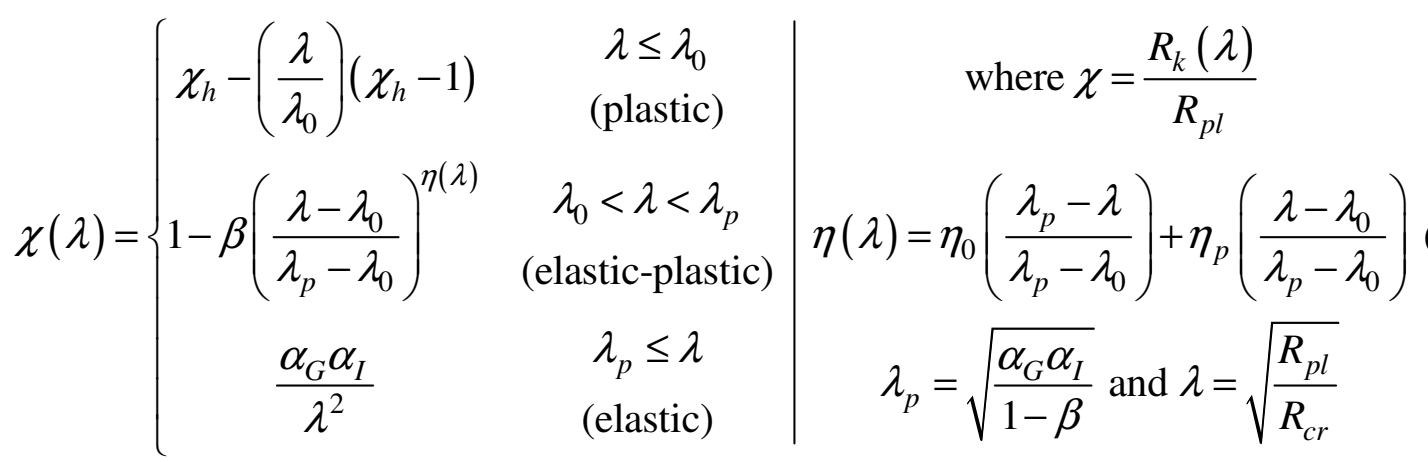

In the above, the product $\alpha=\alpha_{G} \times \alpha_{I}$ gives the 'elastic reduction factor' which accounts for the combined effects of geometric nonlinearity and imperfection sensitivity respectively. By definition, $\alpha_{I}=1$ for perfect shells. The term 'reduction' is slightly misleading and is used for the common understanding in shells, but both effects may be beneficial as well as detrimental depending on the system [18]. The 'plastic range factor' $\beta$ identifies the onset 
of the resistance range over which inelastic behaviour significantly affects the strength, namely for all slendernesses below $\lambda_{p}$. For circular hollow sections (CHS) the slenderness $\lambda_{p}$ corresponds to the boundary between Class 3 'noncompact' and Class 4 'slender' sections. The 'squash limit' slenderness $\lambda_{0}$ identifies the value at which the full plastic moment $M_{p l}$ is just achieved (boundary between Class 2 'compact' to Class 3 'noncompact'). The functional form of the traditional capacity curve (Fig. 1a) is designed to be concave upwards or convex in the elastic region $\left(\lambda \geq \lambda_{p}\right)$, linear in the plastic region (between $(\lambda, \chi)=\left(0, \chi_{h}\right)$ and $\left(\lambda_{0}, 1\right)$, where $\chi_{h}$ is the projected $y$ axis intercept) but may adopt a wide variety of curved shapes in the elastic-plastic region $\left(\lambda_{0}<\lambda<\lambda_{p}\right)$ through an 'interaction exponent' $\eta$ which is currently permitted to vary linearly between boundary values of $\eta_{0}$ and $\eta_{p}$ at $\lambda_{0}$ and $\lambda_{p}$ respectively [16].

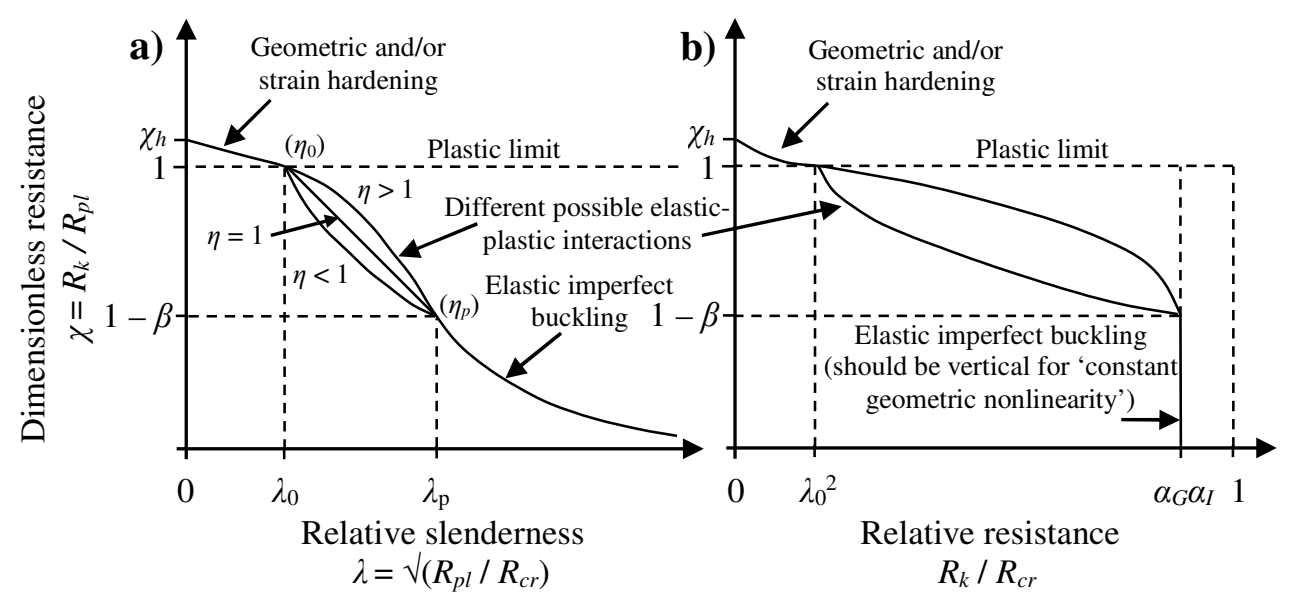

Fig. 1 - a) Traditional dimensionless and b) Modified capacity curves [14].

RRD offers a powerful framework to explore the nonlinear mechanics of structural systems and to translate the outcomes into a compact algebraic form for direct use in manual dimensioning. Because the characterisation is based on mechanical phenomena, sufficient information is contained within the seven independent dimensionless parameters $\alpha_{G}, \alpha_{I}, \beta$, $\eta_{0}, \eta_{p}, \lambda_{0}$ and $\chi_{h}$, together with the two reference resistances $R_{p l}$ and $R_{c r}$, necessary to reproduce a system's behaviour at any slenderness. RRD has now been adopted into the European Standard on Metal Shells EN 1993-1-6 [17,19] through an amendment which additionally created a new normative Annex E to hold sets of RRD parameters for well- 
defined structural configurations and loading. However, the challenge remains to establish a database of these parameters for the most important systems [10], a considerable task for each defined structural problem, since it may require many thousands of individual simulations due to the potentially wide range of geometric, material and loading parameters that govern shell behaviour. A recent paper by Sadowski et al. [20] presented a computational strategy designed to make the programming of vast RRD-oriented parametric analyses manageable for researchers with access to only modest computational resources. This paper offers the first practical application of this methodology, aiming to establish the most comprehensive characterisation to date of elastic linear-hardening metal cylinders under uniform bending.

\section{Length effects under uniform bending}

The geometric nonlinearity of perfect elastic cylindrical shells under uniform bending was explored in the computational study of Rotter et al. [21] who established that the resistance may be described in terms of four domains of behaviour, discriminated by the cylinder length $L$, which must be defined in a dimensionless manner as either $\omega$ or $\Omega$ :

$$
\omega=\frac{L}{\sqrt{r t}} \text { and } \Omega=\frac{L}{r} \sqrt{\frac{t}{r}}
$$

Illustrated in Fig. 2, these length domains are:

- 'Short', where local buckling and ovalisation are fully restrained by the boundary condition and limit point buckling occurs at $M_{k} \gg M_{c r}$ (such that $\left.\alpha_{G} \gg>1\right)$.

- 'Medium', where local short-wave bifurcation buckling occurs adjacent to the most compressed meridian at $M_{k} \approx 0.9 M_{c r}\left(\alpha_{G} \approx 0.9\right)$. The $\sim 10 \%$ reduction from $M_{c r}$ is caused by pre-buckling amplification of the edge boundary condition, as is also seen under axial compression [22]. The resistance in this domain may be characterised as relatively independent of $r / t$, because ovalisation is restrained by the edge boundary conditions when the dimensionless length $\omega$ is less than $0.5(r / t)$, or $\Omega$ is less than 0.5 .

- 'Transitional', where increasing length permits progressively more ovalisation and causes an increasingly nonlinear pre-buckling equilibrium path, leading to a loss of stiffness and premature local buckling on the flattened compressed meridian at $M_{k}$ 
$\ll 0.9 M_{c r}\left(\alpha_{G}<<0.9\right)$. A different dimensionless group $\Omega$ (Eq. $\left.3 b\right)$ is used to characterise this domain, which initiates at $\Omega=0.5$ or $\omega=0.5(r / t)$.

- 'Long', where ovalisation has developed fully and cylinders of all lengths $\Omega \geq 7$ bifurcate elastically at an asymptotic moment $M_{k} \approx 0.5 M_{c r}\left(\alpha_{G} \approx 0.5\right)$.

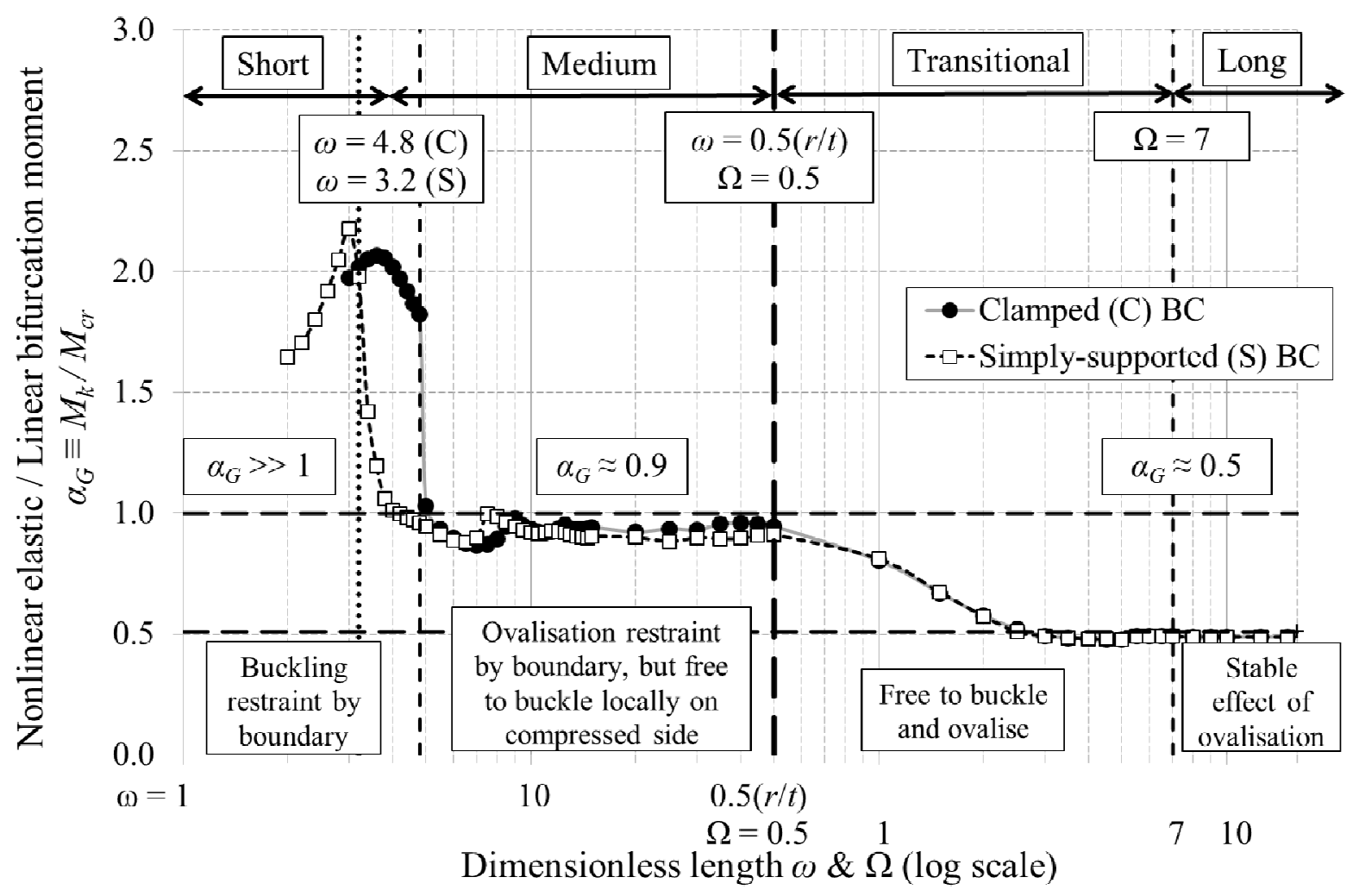

Fig. 2 - Definition of the four length domains governing geometric nonlinearity in perfect elastic cylinders under uniform bending [23].

The dimensionless lengths $\Omega=0.5$ and 7 that define the boundaries between the 'medium' and 'transitional' and between the 'transitional' and 'long' domains respectively are retained here, where the effects of the other system nonlinearities, namely plasticity and imperfection sensitivity, are explored. The 'short' domain and its boundary with the 'medium' domain were the subject of recent detailed studies by Fajuyitan et al. [23-25] who showed that local bifurcation is fully restrained when the cylinder length is of the order of the length of the 'boundary layer' of local compatibility bending. In place of bifurcation, these very short cylinders exhibit a softening load-displacement path leading to 
limit point buckling at a high moment through a hitherto-undocumented 'meridional folding' mechanism, which is insensitive to initial geometric imperfections and may indeed benefit from them. Fajuyitan et al. [23] also studied the differences between pinned and clamped edge rotational restraint conditions on the length of the 'short' domain, finding that the 'medium' domain begins at shorter lengths for pinned edges (unrestrained rotations) than for clamped edges (restrained rotations).

As the 'short' domain represents exceptionally short shells that are of limited practical application, it is not considered further here. Instead, for the purposes of a more compact and conservative characterisation, the 'medium' domain is treated as if it covered the entire length range of non-ovalising cylinders. This simplification also removes the need to include the edge rotational restraint in the characterisation, permitting the two reference resistances to be expressed only in terms of length-independent classical formulae (Eqs 1a and b). Further, as the 'long' domain has been shown to represent 'asymptotic' behaviour that is largely invariant with increasing length [21], it is sufficient to characterise it with a single 'asymptotic' set of RRD parameters obtained at the upper extreme of the 'transitional' domain $(\Omega=7)$. Lastly, the 'modified' capacity curve (Fig. 1b) in $R_{k} / R_{p l}$ vs $R_{k} / R_{c r}$ space exhibits a vertical elastic portion only if the reduction factors due to geometric nonlinearity $\left(\alpha_{G}\right)$ and imperfection sensitivity $\left(\alpha_{I}\right)$ are invariant with slenderness [10,13]. Establishing capacity curves at 'constant geometric nonlinearity' by grouping results at chosen values of an appropriately-constructed dimensionless geometric group is vital, as it reduces the dependencies of the RRD parameters on the geometric variables governing the system [20].

\section{Parameter ranges and modelling methodology}

\subsection{General treatment}

A recent open-access paper by Sadowski et al. [20] presented a detailed strategy illustrating the design and management of a programme of computational analyses aimed at establishing a RRD characterisation of any metal shell system. A central feature is the establishment of an analysis hierarchy as shown in Fig. 3 which arranges the system variables into 'Levels' and establishes their influence on the RRD parameters $\alpha_{G}, \alpha_{I}, \beta, \eta_{0}$, $\eta_{p}, \lambda_{0}$ and $\chi_{h}$. Whist the description given here is expressed in terms of a bi-linear elastic- 
hardening strength relationship, the procedure is not restricted to this material treatment. The Level 0 or 'base state' system variables are the elastic modulus $E$ and yield stress $\sigma_{y}$, set here to $200 \mathrm{GPa}$ and $460 \mathrm{MPa}$ respectively for the entire characterisation. These are 'reasonable' values adopted solely for the purposes of computation, and since the reference resistances $M_{p l}$ and $M_{c r}$ are linear in these two parameters (Eqs 1a and b), the dimensionless RRD formulation is scalable to other elastic stiffnesses and materials. The Level 1 system variable is taken here as the ratio of the post-yield linear strain hardening modulus $E_{h}$ to the elastic modulus $E$, or $E_{h} / E$, assuming a simple bilinear material law. This ratio is set to $0.1 \%$ as a conservative lower bound on strain hardening that supports reasonable practical values of the squash limit $\lambda_{0}[26]$ and the exponent $\eta_{0}$. The Level 2 variable is taken as the normalised amplitude $\delta / t$ of the system imperfection and affects all RRD parameters except $\alpha_{G}$. The system imperfection is the 'Type A' weld depression of Rotter and Teng [27], of which more details are given shortly. The Level 3 and 4 variables are the $r / t$ and $L / r$ ratios respectively, which may be combined into a dimensionless Level 3/4 'length' variable $\omega$ or $\Omega$ (Eq. 3) which, potentially, all RRD parameters are dependent on. A unit wall thickness of $t=1$ was adopted throughout, making all calculations dimensionless.

Table 1 - Computational analyses used and their acronyms [19].

\begin{tabular}{|c|c|c|c|c|c|c|}
\hline Acronym & LBA & MNA & MNIA $\dagger$ & GNA & GNIA & GMNIA \\
\hline $\begin{array}{c}\text { Geometric } \\
\text { nonlinearity }\end{array}$ & No & No & No & Yes & Yes & Yes \\
\hline $\begin{array}{c}\text { Material } \\
\text { nonlinearity }\end{array}$ & No & Yes & Yes & No & No & Yes \\
\hline Imperfections & No & No & Yes & No & Yes & Yes \\
\hline
\end{tabular}

$\dagger$ not strictly an analysis defined in EN 1993-1-6 [19], this is the geometrically linear and materially nonlinear analysis of the imperfect system - see Section 5 for details.

In general, the two reference resistances $M_{p l}$ and $M_{c r}$ are established using parametric LBAs and MNAs (see Table 1) performed at Level 3 (i.e. by varying Level 3 variables, with lower-level variables either kept constant or 'deactivated'). However, this is not necessary for cylinders under bending, as accurate classical closed-form expressions 
already exist for these resistances (Eq. 1; Fig. 2) if the 'short' length domain is excluded. For Level 1 GMNIAs, the $r / t$ and $L / r$ variable ranges were designed to obtain capacity curves at 14 fixed values of $\omega$ between 5 (start of 'medium' domain for all $r / t$ ) and 350 (end of 'medium' domain for $r / t=700$, the thinnest modelled shell), and 20 fixed values of $\Omega$ between 0.5 (start of 'transitional' domain) and 10 (beyond the end of 'transitional' domain and well into the 'long' domain of asymptotic behaviour). A reduced set of Level 2 GNIAs was also performed to establish accurate estimates of the slenderness at which plasticity first substantially affects the resistance, expressed through the $\beta$ parameter. An additional set of Level 2-3 MNIAs was performed to establish the reference plastic collapse resistance $M_{p l, i m p}$ of imperfect cylinders. Each analysis was generated, submitted, terminated and processed without repetitive human intervention using the automated methodology of Sadowski et al. [20] at around 700 unsupervised analyses per day on two computer workstations. The parameter ranges are shown in Table 2.

Table 2 - Parameter ranges and numbers of individual computational analyses.

\begin{tabular}{|c|c|c|c|c|c|}
\hline Set & Level 1 $\left(E_{h}\right)$ & Level 2 $(\delta / t)$ & Level 3 $(r / \mathrm{t})$ & Level 4 $(L / r)$ & $\begin{array}{c}\text { Total no. of } \\
\text { analyses }\end{array}$ \\
\hline GMNIA & $0.1 \%$ of $E(\mathbf{1})$ & $0.01-3(\mathbf{1 0})$ & $10-700(\mathbf{5 8})$ & $\begin{array}{c}\omega=5-350 ; \Omega \\
=0.5-10(\mathbf{3 1})\end{array}$ & $\begin{array}{c}1 \times 10 \times 58 \times 31 \\
=17,980\end{array}$ \\
\hline GNIA & $n / a$ & $0.01-3(\mathbf{1 0})$ & $100-500(\mathbf{3 2})$ & $\begin{array}{c}\omega=5-350 ; \Omega \\
=0.5-10(\mathbf{3 1})\end{array}$ & $\begin{array}{c}10 \times 32 \times 31 \\
=9,920\end{array}$ \\
\hline MNIA & $n / a$ & $0.01-3(\mathbf{1 0})$ & $10-700(\mathbf{3 5})$ & $n / a$ & $10 \times 35=350$ \\
\cline { 5 - 6 } & \multicolumn{3}{|r}{} & Total no. & 28,250 \\
\hline
\end{tabular}

\subsection{Imperfection form}

The axisymmetric 'Type A' circumferential weld depression of Rotter and Teng [27] was used as the imperfection form throughout this study, with a single instance placed at midspan. Defined by a single meridional profile (Eq. 4), it is an analytical idealisation of the inward curling of the shell wall caused by both the anticlastic bending of the edges of rolled shell plates and the shrinking of a cooling weld. It has been shown by calibration on surface imperfection surveys to be a realistic representation of this systematic manufacturing defect [28-33], and has been extensively used over the past 30 years in 
numerical studies of imperfection sensitivity in cylindrical shells, especially where meridional compressive stresses initiate buckling under various load conditions (in December 2019, the Scopus system showed 142 citations of Rotter and Teng [27]). The companion study by Fajuyitan and Sadowski [34] had additionally shown that the weld depression is consistently more deleterious for cylinders under bending than the classical linear buckling eigenmode or ovalisation harmonic imperfections.

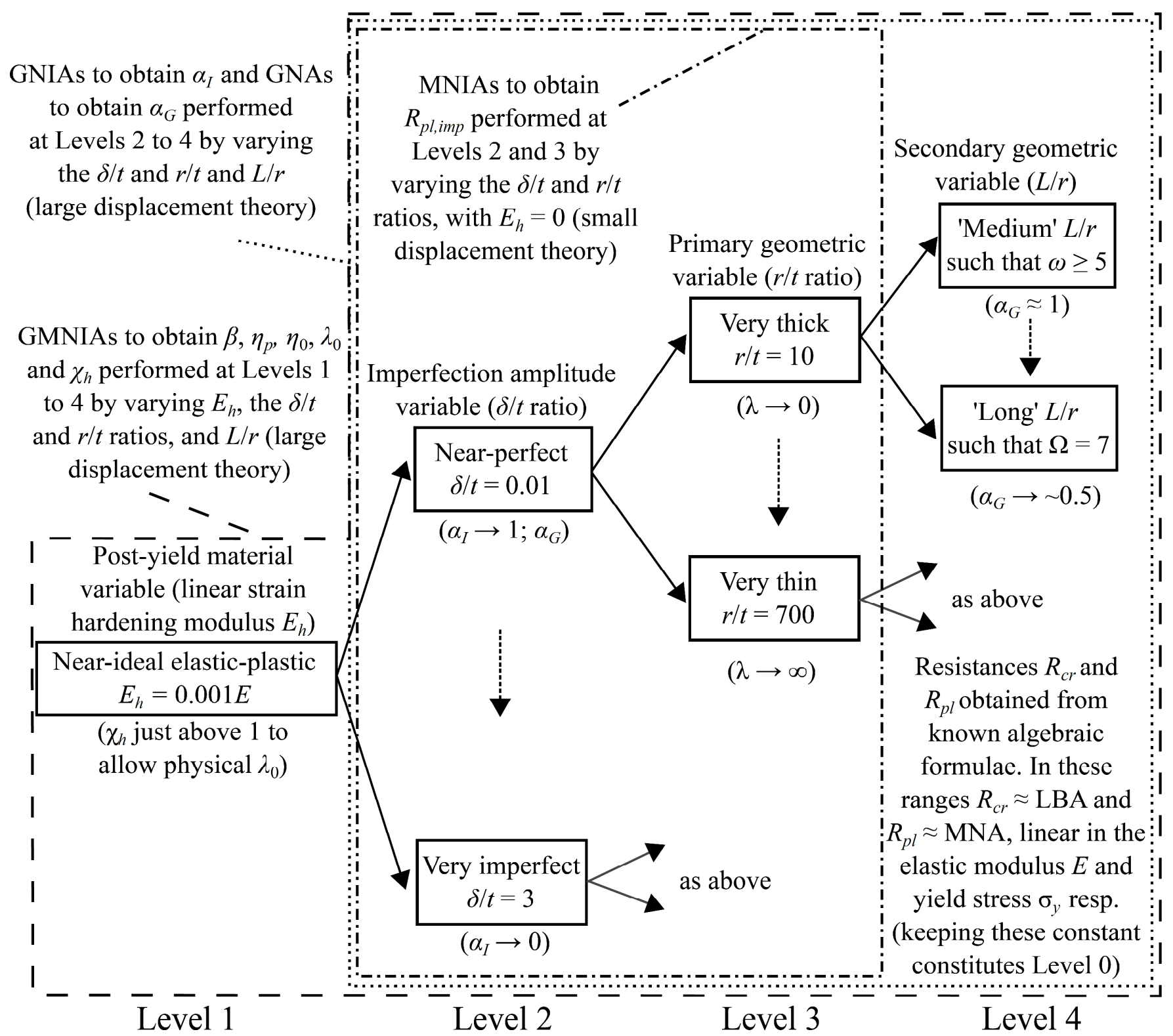

Fig. 3 - Specialised RRD analysis hierarchy for isotropic metal cylinders under uniform bending (after Sadowski et al. [20]). 
For each combination of the $r / t$ and $L / r$ variables, the imperfection amplitude $\delta / t$ was varied from 0.01 or 0.001 (here termed 'near perfect') to 3 (deemed very imperfect). A weld depression located at midspan (meridional coordinate of $z=L / 2$ ) is defined by:

$$
w(z)=\delta e^{-\frac{\pi}{\Lambda}\left|z-\frac{L}{2}\right|}\left[\cos \left(\frac{\pi}{\Lambda}\left|z-\frac{L}{2}\right|\right)+\sin \left(\frac{\pi}{\Lambda}\left|z-\frac{L}{2}\right|\right)\right]
$$

where $\Lambda \approx 2.444 \sqrt{ }(r t)$ is the linear meridional bending half-wavelength.

\subsection{Finite element model}

The template for each finite element model is shown in Fig. 4 and follows the same design as in the authors' previous studies [21,23-25,35,36]. Using the ABAQUS [37] software, a quarter of the shell was modelled for computational efficiency with appropriate use of two sets of symmetry boundary conditions. The meridional mesh resolution was carefully refined throughout the zones within $3 \Lambda$ of the loaded end boundary and within $3 \Lambda$ of the midspan weld depression imperfection to capture local bending effects. Where these two regions overlapped for shorter medium-length cylinders (but still $\omega>5$ ), a uniform element spacing was applied meridionally. The circumferential element size was graded to produce a fine mesh of approximately square elements on the compression side but a courser mesh on the tension side. The general-purpose reduced-integration four-node S4R shell element was used throughout.

These ABAQUS models were previously validated by Sadowski and Rotter [35] for tubes as thick as $r / t=10$ under combined high strains and cross-sectional ovalisation. Both the experimental and numerical moment-curvature and ovalisation-curvature relationships of Kyriakides and Ju [38,39] were accurately reproduced. Similar ABAQUS models were successfully used by Vasilikis et al. [40] to model experiments described by van Es et al. [8] on the elastic-plastic buckling of thick ( $r / t$ varying from 32 to 64) spiral-welded steel tubes under bending. The power of the Riks [41] arc-length solver in ABAQUS to model elastic bifurcation and mode-switching in thin cylindrical shells $(r / t \approx 400)$ was illustrated by Kobayashi et al. [42], who reproduced load-displacement paths of laboratory-tested cylinders under uniform compression [22,43] with a high degree of fidelity. Finally, Rotter et al. [21] had shown that ABAQUS predicts an elastic ovalisation response in cylinders of 
finite length under bending consistent with predictions by algebraic and numerical studies that did not use the finite element method, and tends to the limiting Brazier [1] reference values for asymptotically long cylinders at all $r / t$. Each of the above studies used the S4R element.

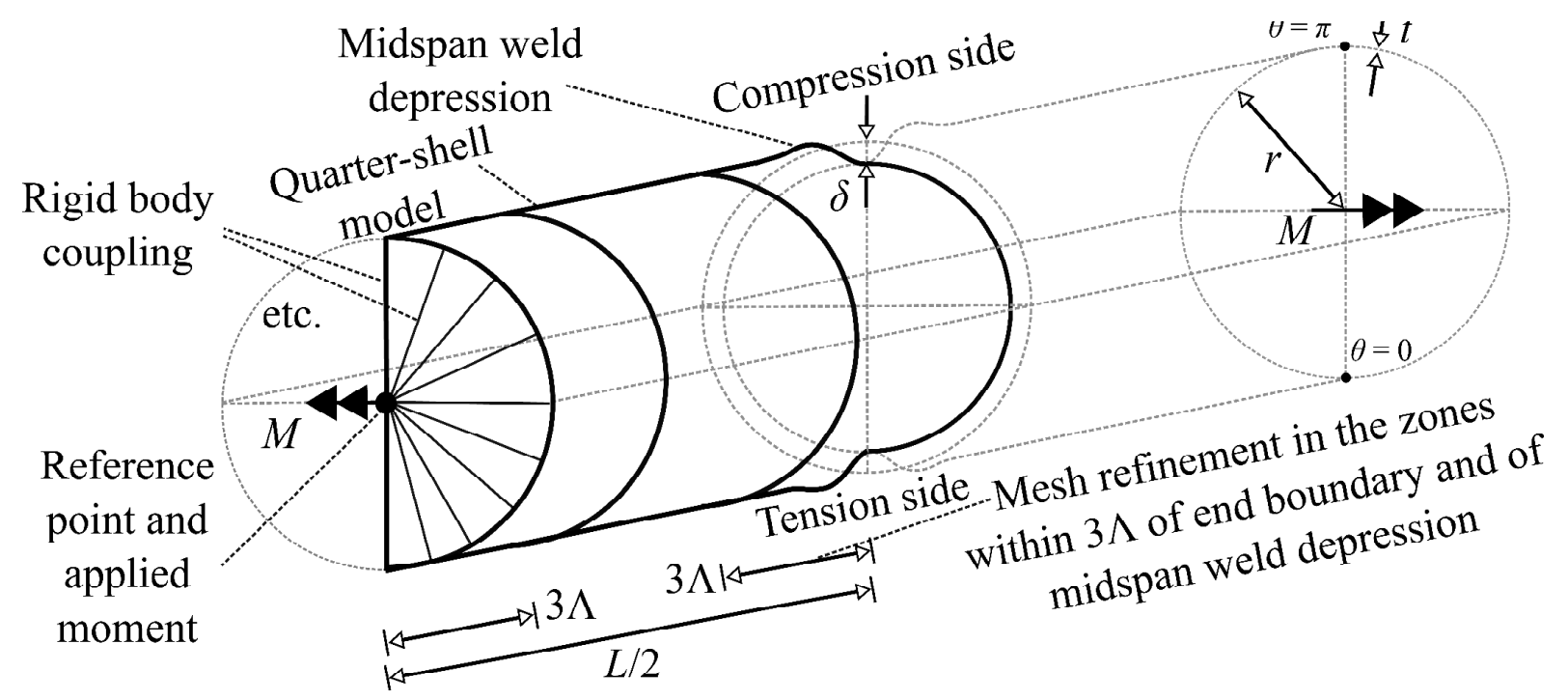

Fig. 4 - Template for each finite element model.

\section{Reference full plastic moment resistance for cylinders with midspan weld depression imperfections}

\section{$5.1 \quad$ Introduction}

The two linear reference moments $M_{p l}$ and $M_{c r}$ are the normalising parameters of the RRD characterisation and must be established as accurately as possible. However, the current RRD functional form (Eq. 2) becomes ill-suited to express capacity curves of very imperfect cylinders at low cross-section slenderness if it is always related to the full plastic moment of the perfect cylinder $M_{p l}$. Deeper imperfections depress the capacity curve to the extent that a characterisation in terms of $M_{p l}$ leads to non-physical negative values of the squash limit slenderness $\lambda_{0}$ (illustrated schematically in Fig. 5a). This arises because the systematic treatment in terms of fixed imperfection amplitudes, to give total generalisation, leads to the requirement to include deep imperfections in stocky structures. The key reason why this retention of fixed imperfection amplitudes is so important is that the elasticplastic parameters $\eta_{0}, \lambda_{0}$ and $\chi_{h}$ are significantly affected by the treatment of fully plastic conditions, so a generalisation to free the description of assumptions concerning the 
imperfection amplitudes that may occur demands that deep imperfections are carried all the way to fully stocky structures. This problem can also be seen in the calculations on axial compression of Rotter [44]. It was suggested in Rotter [12] that it is 'reasonable to stop the calculations at perhaps $M_{k} / M_{p l} \sim 0.9$ and not to try to obtain values of $\lambda_{0}$ for deep imperfections' since such deep imperfections do not arise in shells susceptible to heavy plasticity (p. 9). However, this proposed cut-off is quite arbitrary and makes it difficult to build full parameter models that can be automated. An alternative approach is to ignore strain hardening which ensures that the full-plastic condition can never be attained. This then forces $\lambda_{0}$ to always be zero [45], but such a treatment is inconsistent with documented test results which show that the full plastic condition is easily attained in perfect cylinders under both axial compression and bending at finite slendernesses [26,46,47]. It may be noted that these test results also naturally depend on the real imperfection forms and amplitudes.
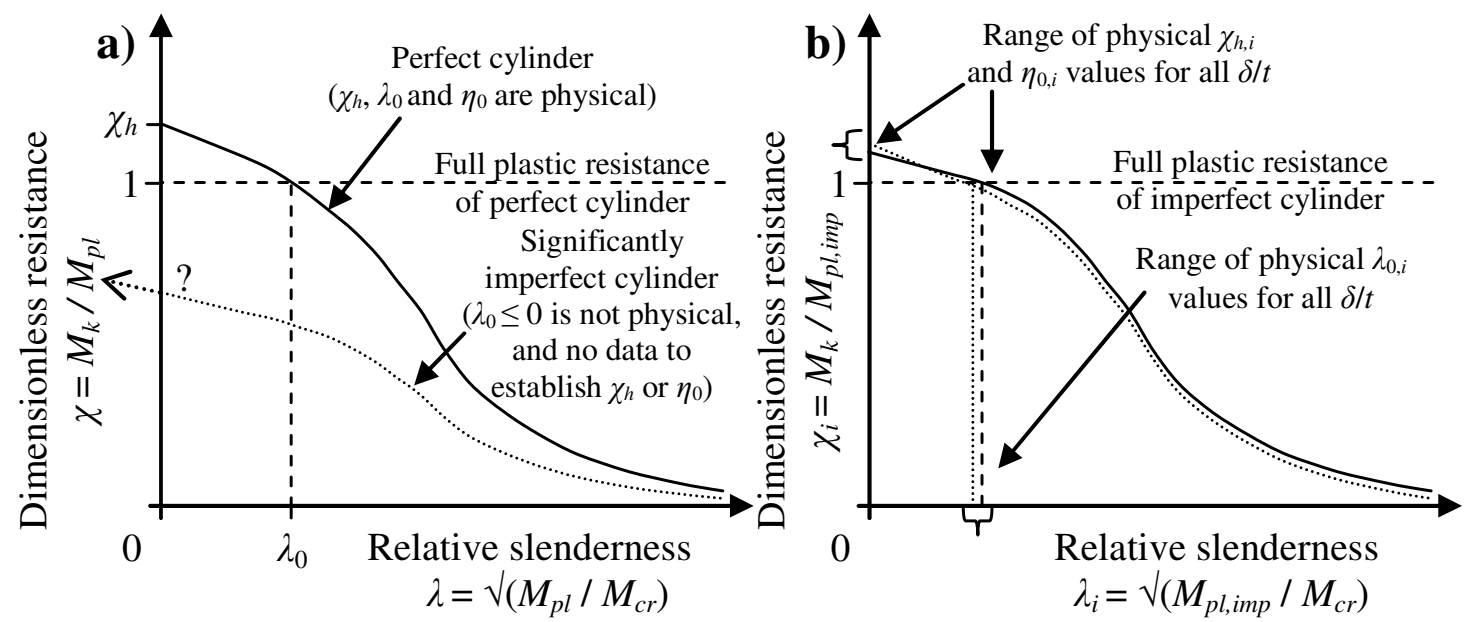

Fig. 5 - Proposed modification to the RRD base relationship to allow a more realistic characterisation of plasticity in imperfect cylinders.

Instead of the above ad hoc treatments, the RRD formulation is here modified by replacing $M_{p l}$ with $M_{p l, i m p}$, the full plastic moment of an imperfect cylinder assuming the same imperfection form as in the accompanying GMNIA analyses, with a single midspan weld depression (Eq. 4) and making $M_{p l, i m p}$ dependent on the imperfection amplitude. The relative slenderness is then related to $M_{p l, i m p}$. This treatment has the benefit of permitting 
the plasticity-related RRD parameters $\chi_{h}, \lambda_{0}, \eta_{0}, \eta_{p}$ and $\beta$ (including $\lambda_{p}$ ) to retain their direct physical meaning for imperfect systems (where they now adopt the subscript ' $i$ ' to become $\chi_{h, i}, \lambda_{0, i}, \eta_{0, i}, \eta_{p, i}$ and $\beta_{i}$ ), resolving a recurrent difficulty and amplifying the predictive power of the RRD framework (Fig. 5b). The elastic-only RRD parameters $\alpha_{G}$ and $\alpha_{I}$ remain unchanged from their original definitions. The revised formulation then becomes:

$$
\chi_{i}\left(\lambda_{i}\right)=\left\{\begin{array}{cc|c}
\chi_{h, i}-\left(\frac{\lambda_{i}}{\lambda_{0, i}}\right)\left(\chi_{h, i}-1\right) & \begin{array}{c}
\lambda_{i} \leq \lambda_{0, i} \\
\text { (plastic) }
\end{array} \\
1-\beta_{i}\left(\frac{\lambda_{i}-\lambda_{0, i}}{\lambda_{p, i}-\lambda_{0, i}}\right)^{\eta_{i}\left(\lambda_{i}\right)} & \begin{array}{c}
\lambda_{0, i}<\lambda_{i}<\lambda_{p, i} \\
(\text { elastic-plastic) }
\end{array} & \eta_{i}\left(\lambda_{i}\right)=\eta_{0, i}\left(\frac{\lambda_{p, i}-\lambda_{i}}{\lambda_{p, i}-\lambda_{0, i}}\right)+\eta_{p}\left(\frac{\lambda_{i}-\lambda_{0, i}}{\lambda_{p, i}-\lambda_{0, i}}\right) \\
\frac{\alpha_{G} \alpha_{I}}{\lambda_{i}^{2}} & \begin{array}{c}
\lambda_{p, i} \leq \lambda_{i} \\
\text { (elastic) }
\end{array} & \lambda_{p, i}=\sqrt{\frac{\alpha_{G} \alpha_{I}}{1-\beta_{i}}} \text { and } \lambda_{i}=\sqrt{\frac{\kappa R_{p l}}{R_{c r}}}
\end{array}\right.
$$

The above reduces to the original formulation in Eq. 2 when $\delta / t=0$ as $\kappa=R_{p l, i m p} / R_{p l}=1$. In the following section, a closed-form empirical expression for the $M_{p l \text {,imp }}$ reference resistance is derived, based on the results of a series of computational MNIA analyses.

\subsection{Computational MNIA analyses}

A series of computational MNIA analyses was carried out to establish an empirical algebraic expression for the reference full plastic moments of imperfect cylinders $M_{p l \text {,imp }}$. A preliminary set of MNIA models with constant $r / t=100$ and $\delta / t=2.0$ was analysed for four different dimensionless lengths $(\Omega=0.5,1,2$ and 3; all long enough for the midspan weld depression to be unaffected by end boundary conditions) and under two representative load arrangements ('two-point' and 'four-point' bending arrangements). The calculated relationships between moment and plastic hinge rotation are shown in Fig. 6, where the data indicates that neither the length nor the loading arrangement has any significant effect on the equilibrium curves at constant $\delta / t$ and $r / t$. This limits the control parameters to only the Level $2(\delta / t)$ and Level $3(r / t)$ system variables for the remaining MNIA investigations, which also use the simpler 'two-point' loading arrangement. 


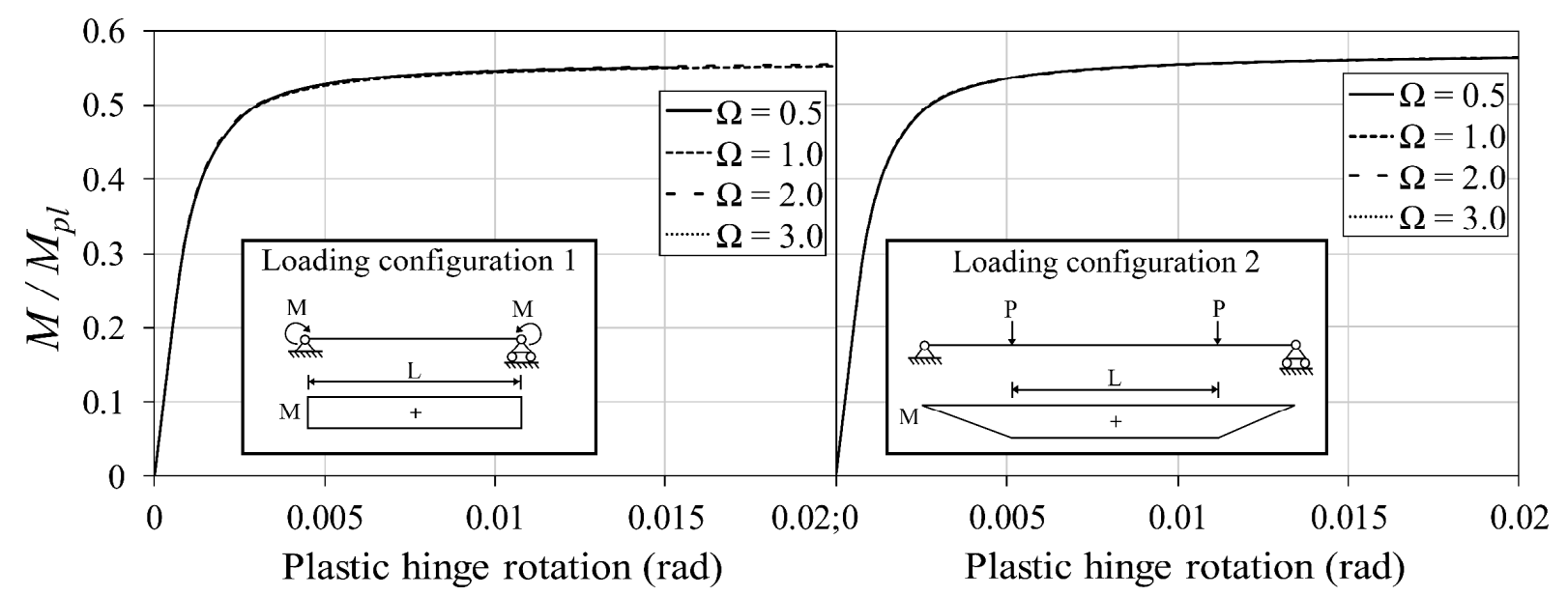

Fig. 6 - Equivalence of MNIA equilibrium curves of moment against plastic hinge rotation (full end rotation minus rotation due to elastic deformation) for two loading configurations for dimensionless lengths free of boundary effects, $r / t=100$ and $\delta / t=2$.

A total of 350 MNIAs were performed next at Levels 2 and 3, with $\delta / t$ varied from 0.01 to 3 and $r / t$ from 10 to 700 (Table 2; Fig. 3), all at a single representative dimensionless length of $\Omega=0.5$. Since the plastic plateau on a moment-rotation curve using MNIA may be slow or numerically problematic for systems with plastic collapse mechanisms that involve extensive local bending and surface yielding, the 'Convergence Indicator Plot' method devised by Doerich and Rotter [16] and implemented for automation in ABAQUS by Sadowski et al. [20], was employed. This allows an accurate estimate of the plastic collapse moment to be found, based only on a partially-computed portion of a still-rising equilibrium curve. The computed $M_{p l, i m p}$ values were normalised by the full plastic moment resistance of perfect shells $M_{p l}$ calculated using the finite-thickness expression in Eq. 1a, and are plotted in Fig. 7 against $r / t$ for different imperfection amplitudes $\delta / t$. A selection of 'plastic imperfection sensitivity' relationships were additionally obtained (Fig. 8) for constant $r / t$ but varying $\delta / t$. It is evident that small imperfection amplitudes are much less detrimental to the plastic reference resistance than they are to the elastic buckling resistance under meridional compression. 


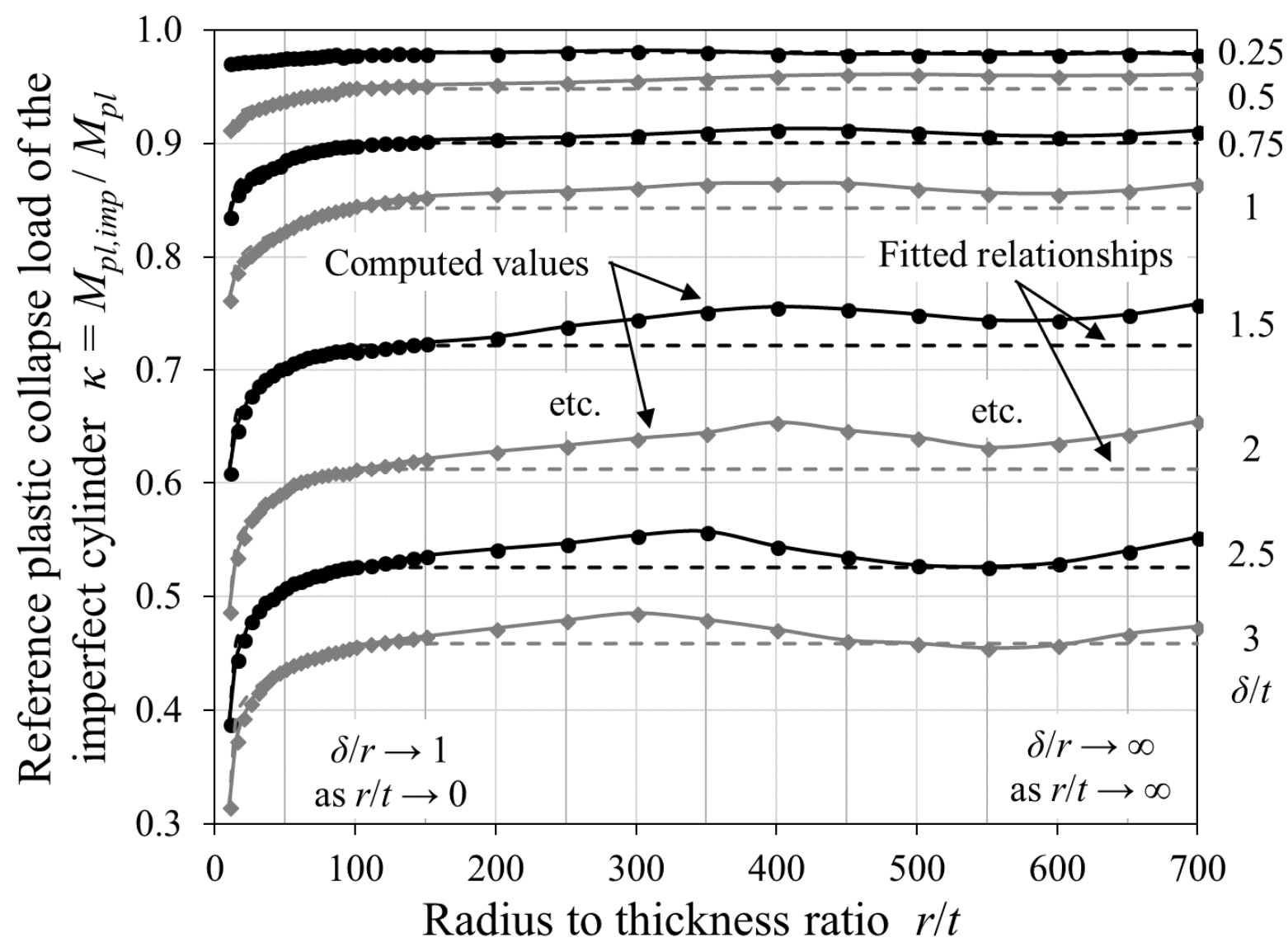

Fig. 7 - Variation of the $\kappa=M_{p l, i m p} / M_{p l}$ 'plastic imperfection sensitivity' relationship with $r / t$ and $\delta / t$ for cylinders of length $\Omega=0.5$ with the weld depression imperfection.

The 'plastic imperfection sensitivity' established in this manner is seen to be more severe for thicker cylinders (Fig. 8), as an imperfection with an amplitude of a single wall thickness represents a deviation covering a significant portion of the radius in a thick cylinder but only a negligible portion of the radius in a thin cylinder. (e.g. $\delta / t=1$ is $10 \%$ of the radius when $r / t=10$ but only $1 \%$ when $r / t=100$ ). An attenuation of the severity of the sensitivity is observed for thinner cylinders, and beyond $r / t \approx 100$ the relationship is largely invariant with $r / t$. It should be added that while the weld depression is a credible imperfection in thin shells where the tolerances are difficult to control during fabrication, in thicker tubes it is often of less certain relevance. However, it remains a valuable and consistent modelling device to investigate imperfection sensitivity across all slendernesses. 




Fig. 8 - Individual $\kappa=M_{p l, i m p} / M_{p l}$ 'plastic imperfection sensitivity' relationships at different $r / t$ for cylinders of length $\Omega=0.5$ with the weld depression imperfection.

\subsection{Algebraic characterisation}

A generalised power law functional form was adopted to characterise the plastic reference resistance for imperfect cylinders, drawing on functional forms widely used in the past for cylinders under various fundamental loads $[19,44,45,48]$. As thin cylinders with $r / t>\sim 100$ achieve a relatively stable 'plastic imperfection sensitivity' (Fig. 7), they may be described by a conservative lower bound characterisation as follows:

$$
\kappa_{\text {thin }}=0.2+\frac{0.8}{1+\left[0.014(\delta / t)^{0.2}+0.23(\delta / t)^{2}\right]} \text { for } r / t \geq 100
$$

An adjustment is necessary for thicker cylinders due to the increasing size of the imperfection relative to the total radius. The relationship for the thickest practical shell with $r / t=10$ may be expressed as: 
$\kappa_{\text {thick }}=\frac{1}{1+\left[0.093(\delta / t)^{1.3}+0.222(\delta / t)^{1.9}\right]}$ for $r / t=10$

The complete relationship is now as follows, with intermediate thicknesses may be obtained by simple interpolation:

$$
\kappa=\left\{\begin{array}{cc}
\kappa_{\text {thick }} & r / t=10 \\
\kappa_{\text {thick }}+\left(\kappa_{\text {thin }}-\kappa_{\text {thick }}\right)\left[\frac{r / t-10}{90}\right]^{0.35-0.05(\delta / t)} & 10<r / t<100 \\
\kappa_{\text {thin }} & r / t \geq 100
\end{array}\right.
$$

The predictions of the above equations are compared against the computed FE values in Figs 7 and 8 and indicate a conservative but representative algebraic representation.

\section{Computed capacity curves in the 'medium' length domain}

\subsection{Introduction}

A small selection of the extensive number of GMNIA calculations performed for cylinders under bending in the 'medium' length domain $(5.0 \leq \omega \leq 0.5 r / t)$ are presented in this section in the form of modified capacity curves (Fig. 1b) in $M_{k} / M_{p l}$ vs $M_{k} / M_{c r}$ space (Fig. 9). Due to space limitations, only capacity curves at constant dimensionless lengths $\omega=5$ (boundary with the 'short' domain), 10, 50, $100 \& 350$ (boundary with the 'transitional' domain for the thinnest analysed shell with $r / t=700$ ) and constant imperfection amplitudes $\delta / t=0.01$ ('near perfect'), $0.1,0.5,1.0 \& 2.0$ are shown here, with varying $r / t$ to change the relative slenderness. The use of a small 'perturbation' of $\delta / t=0.01$ (initially) for 'near perfect' cylinders was to ensure numerical stability, as it was not possible to achieve convergence for a nominally perfect geometry with no mesh perturbation of any kind. The 'modified' capacity curves allow the three regions illustrated in Fig. 1b to be identified: an elastic region that is manifest as a nearly vertical line at the right side of every curve in $M_{k}$ $/ M_{p l}$ vs $M_{k} / M_{p l}$ space, a curved elastic-plastic region in the middle, and a strain-hardening region exceeding $M_{p l}$ at the left side of every 'near perfect' curve. 


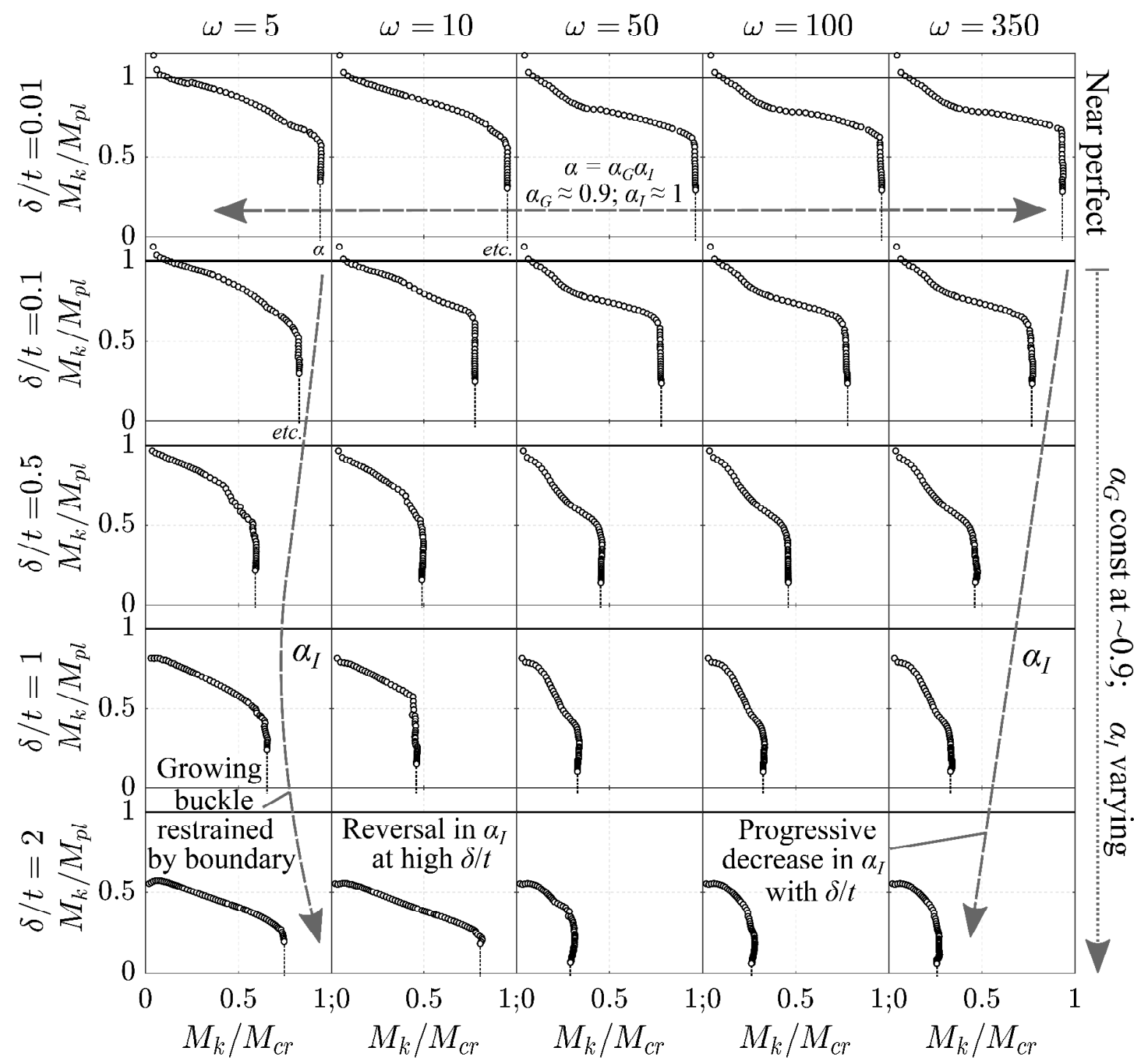

Fig. 9 - A selection of modified capacity curves in $M_{k} / M_{p l}$ vs $M_{k} / M_{c r}$ space at constant $\delta / t$ and $\omega$ within the 'medium' length domain ( $\delta / t=0.01$ for 'near perfect' cylinders).

\subsection{The elastic portion of the capacity curve}

The elastic region may be identified as a distinct near-vertical line on the right-hand side of each curve that intercepts with $M_{k} / M_{c r}$ at a value of $\alpha=\alpha_{G} \alpha_{I}$. Minor variations due to numerical nature of the predictions aside, these analyses indicate that the dimensionless geometric groups $\omega$ and $\delta / t$ largely preserve invariant geometric nonlinearity at varying $r / t$ for both 'near perfect' and imperfect cylinders in this length domain. The 'near perfect' curves for $\delta / t=0.01$ exhibit an elastic reduction in $M_{k}$ due to geometric nonlinearity of approximately $10 \%$ at all $\omega$, consistent with previous findings for $\alpha_{G} \approx 0.9$ in the 'medium' length domain (Fig. 2; [21]). For cylinders longer than $\omega \approx 10$, the vertical intercept on $M_{k}$ 
$/ M_{c r}$ reduces progressively with increasing $\delta / t$ (decrease in $\alpha_{I}<1$ while $\alpha_{G}$ remains constant at $\sim 0.9$ ), with the imperfection having a consistently deleterious effect. However, for cylinders shorter than $\omega \approx 10$, an increase in $\delta / t$ eventually causes a rise in $\alpha_{I}$. This is caused by the progressive expansion of the buckling mode with imperfection amplitude, which in short cylinders becomes constrained by the end boundary conditions. This phenomenon is well documented elsewhere in the shell buckling literature [22-25,27,49].

\subsection{The 'first yield plateau' in 'near perfect' cylinders of intermediate slenderness}

The shape of the capacity curve for 'near perfect' cylinders in the elastic-plastic region deserves a detailed explanation (Fig. 10), as the phenomenon described here does not appear to have been previously reported or understood. In the first calculations on cylinders with only tiny imperfections (a 'perturbation' amplitude of $\delta / t=0.01$ ), plasticity was found to affect the buckling resistance at a moment approximately $16 \%$ below the first yield moment $M_{e l}$, as calculated by classical beam theory (Eq. 9). This was partly because the GMNAs were found to predict the elastic-plastic buckles to develop within the bending boundary layer (located $3 \Lambda$ from the loaded edge; Fig. 4) where surface yielding caused by compatibility bending acted as a stronger buckling trigger than the tiny weld depression at midspan. The effects of this compatibility bending were eliminated by modelling the nearedge regions with elastic material, only allowing plasticity in the midspan region. Despite this change, plasticity was still found to initiate buckling at a moment $\sim 11 \%$ below $M_{e l}$. This was then attributed to early outer surface yielding at the tiny imperfection, so the amplitude of the weld depression was reduced by an order of magnitude to $\delta / t=0.001$. Plasticity was then found to initiate buckling at only $\sim 4 \%$ below $M_{e l}$ (Figs 10a and b). For cylinders with midspan imperfections deeper than $\delta / t=0.1$, buckling was never found to occur at the edge regions which were then no longer modelled as elastic.

$$
M_{e l}=\left(\frac{4 r^{2}+t^{2}}{4 r+2 t}\right) \pi r t \sigma_{y} \approx \pi r^{2} t \sigma_{y} \text { as } \frac{r}{t} \rightarrow \infty
$$




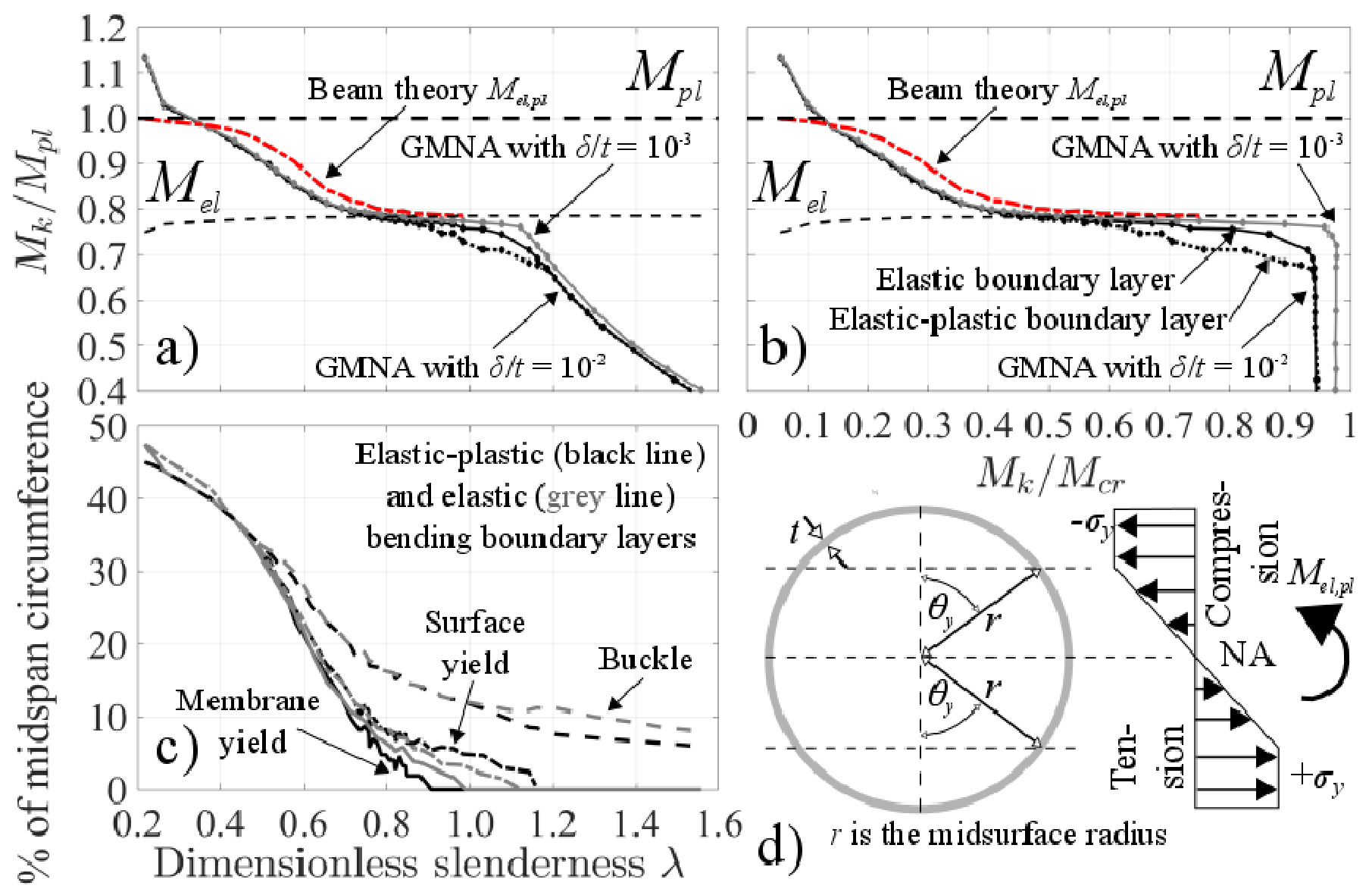

Fig. 10 - Selected data for 'near-perfect' cylinders of 'medium' length $(\omega=350)$.

The onset of surface yielding appears to have an immediate detrimental effect on the bending resistance (Figs 10a and b). With decreasing slenderness, the capacity curves exhibit a distinct 'plateau' at moments close to the first yield value $M_{e l}$. This effect occurs over a wide range of slendernesses, $\sim 0.7<\lambda<\sim 1.15$. The strong interaction between plasticity and local buckling can be explained by considering the relative portions of the circumference covered by the midspan local buckling mode (estimated as the angular distance between the radially innermost to outermost deflections in the incremental buckling mode) and the extent of surface and membrane yielding in its vicinity (Fig. 10c). Under bending, a large portion of the compressed side is under conditions of approximately uniform meridional compression, so that the angular extent of a finite-size midspan buckle is proportional to $\sqrt{ }(r t)$. In turn, the portion of the cylinder circumference that this buckle occupies is proportional to $\sqrt{ }(r t) / r=\sqrt{ }(t / r)$. As the slenderness decreases (reducing $r / t$ ) the buckle occupies a progressively wider proportion of the circumference of 
the shell. The regions of surface and membrane yield at buckling similarly grow progressively with decreasing $r / t$ (Fig. 10c). If the circumferential extent of membrane yield is known, the moment necessary to cause it $M_{e l, p l}$ may be estimated using classical beam theory (treating the cylinder as a 'thin' circular hollow section for simplicity) as:

$$
M_{e l, p l} \approx\left(\frac{2 \cos \theta_{y} \sin \theta_{y}+\pi-2 \theta_{y}}{\pi \cos \theta_{y}}\right) r^{2} t \sigma_{y} \rightarrow\left\{\begin{array}{l}
M_{e l} \approx \pi r^{2} t \sigma_{y} \quad \text { as } \theta_{y} \rightarrow 0 \\
M_{p l} \approx 4 r^{2} t \sigma_{y} \quad \text { as } \theta_{y} \rightarrow \pi / 2
\end{array}\right.
$$

where $\theta_{y}$ is the half-angle spread of a cylinder under membrane yield (Fig. 10d).

Estimating $\theta_{y}$ from GMNAs at buckling (the 'membrane yield' curve in Fig. 10c), the capacity curve deduced on the basis of $M_{e l, p l}$ is shown in red in Figs 10a and b. This capacity curve effectively assumes that the yielded region has no buckling resistance and a buckle forms entirely within it. However, as a real buckle is always partially elastic (Fig. $10 \mathrm{c})$, this red $M_{e l, p l}$ curve must give an upper bound to the computed GMNA curve, while a lower bound must be given by the moment at first yield (slightly below the $M_{e l}$ prediction). A close inspection of Eq. 10 suggests that $M_{e l, p l}$ rises only very slowly with small values of $\theta_{y}$, so that these narrow theoretical bounds force the computed GMNA curve to be almost flat over a wide range of intermediate slendernesses: a yielded region of $10 \%$ of the circumference that corresponds approximately to the lower end of the 'first yield plateau' at $\lambda \approx 0.7$ requires a moment that is only $\sim 4 \%$ above the $M_{e l}$ attained at $\lambda \approx 1.15$. The flattened shape of these capacity curves appears to be unique to members with circular cross-sections under bending.

This shape of this capacity curve has particularly important implications for the design of thicker cylinders under bending. Unlike most other capacity curves, the long plateau close to first yield must lead to a very steep fall in resistance from the full plastic moment to the first yield moment (Fig. 10a). This dramatic fall is not evident in axially compressed cylinders, nor in the buckling of beams and columns. The significantly changed shape of the capacity curve means that the traditional established concept [50] that axially compressed cylinder strengths offer a safe lower bound for all shell buckling conditions may be seriously in error. This important conclusion will be explored by the authors as part of a later study. 


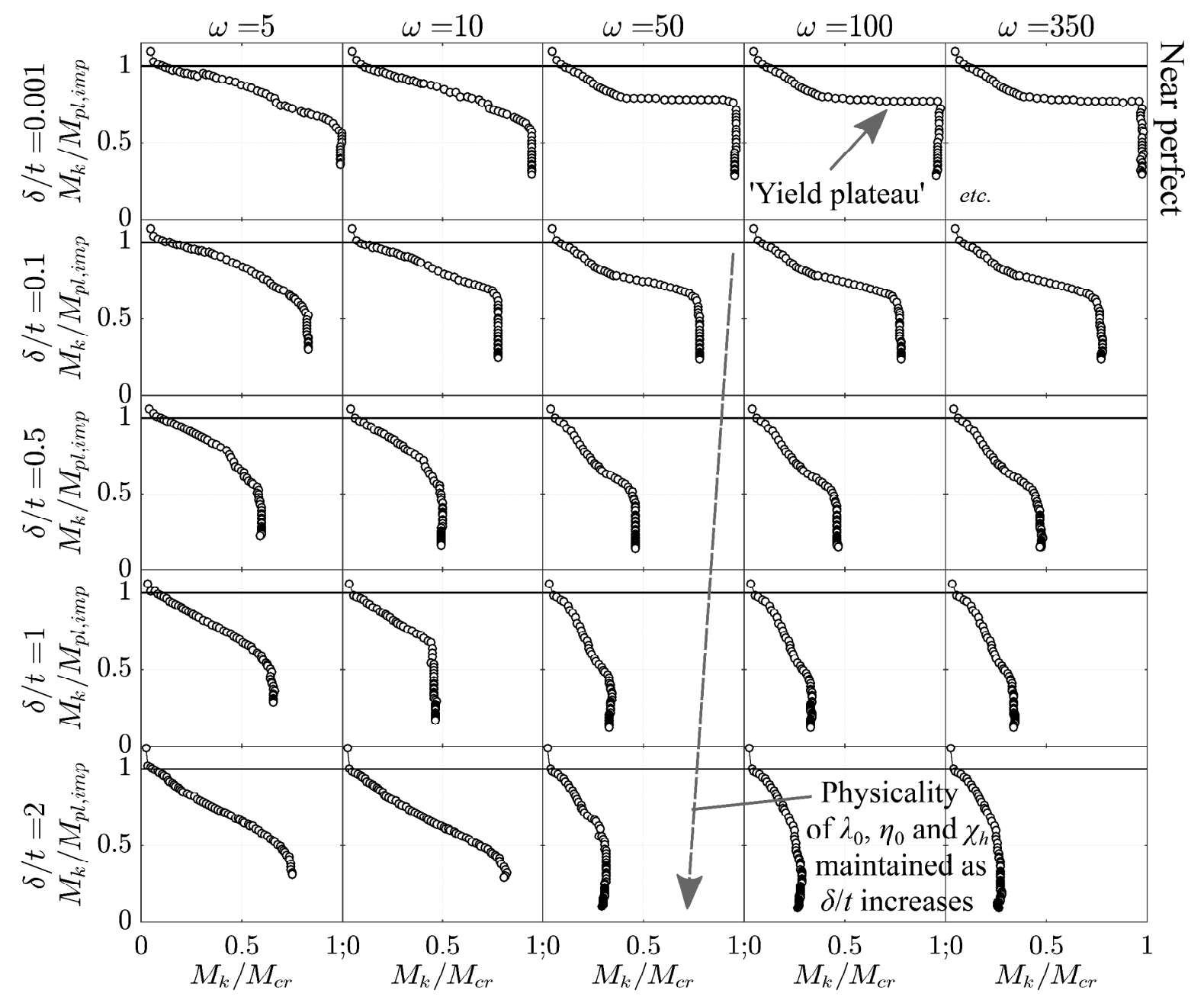

Fig. 11 - A selection of modified capacity curves in $M_{k} / M_{p l, i m p}$ vs $M_{k} / M_{c r}$ space at constant $\delta / t$ and $\omega$ within the 'medium' length domain $(\delta / t=0.001$ for 'near perfect' cylinders).

\subsection{The fully plastic portion of the capacity curves}

The evolution of the left-hand portions of the capacity curves with increasing imperfection amplitude seen in Fig. 9, representing the resistance of fully-plastified thick cylinders, illustrates the need for the modification to the RRD framework proposed in Fig. 5. This portion of the curve becomes increasingly ill-defined at deeper $\delta / t$ and ceases to attain $M_{k}=$ $M_{p l}$ at imperfection greater than $\delta / t \approx 0.5$. It is no longer possible to represent these curves using the original equation of Eq. 2 [14] because the RRD parameters $\lambda_{0}, \eta_{0}$ and $\chi_{h}$ can no longer be identified. The same capacity curves are presented in the alternative form of $M_{k} /$ $M_{p l, i m p}$ vs $M_{k} / M_{c r}$ in Fig. 11 , where $M_{p l, i m p}$ is the computed MNIA prediction of the 
reference plastic resistance of the imperfect cylinder (Fig. 7). This transformation preserves the intended form of the capacity curve, allowing revised RRD parameters $\lambda_{0, i}$, $\eta_{0, i}$ and $\chi_{h, i}$ to retain their physical meaning and significantly simplifying the task of establishing complete sets of characterising RRD parameters. Additionally, the adjusted relationship fully preserves the previous description of the elastic behaviour and the values of the parameters $\alpha_{G}$ and $\alpha_{I}$. The values of $\lambda_{0, i}$ obtained using the amended treatment fall in the range of $\lambda_{0, i}$ from $\sim 0.2$ to $\sim 0.3$, broadly consistent with values obtained from test programmes $[4,46,47]$, with either very long or imperfect cylinders exhibiting $\lambda_{0, i}$ at the lower end of this range.

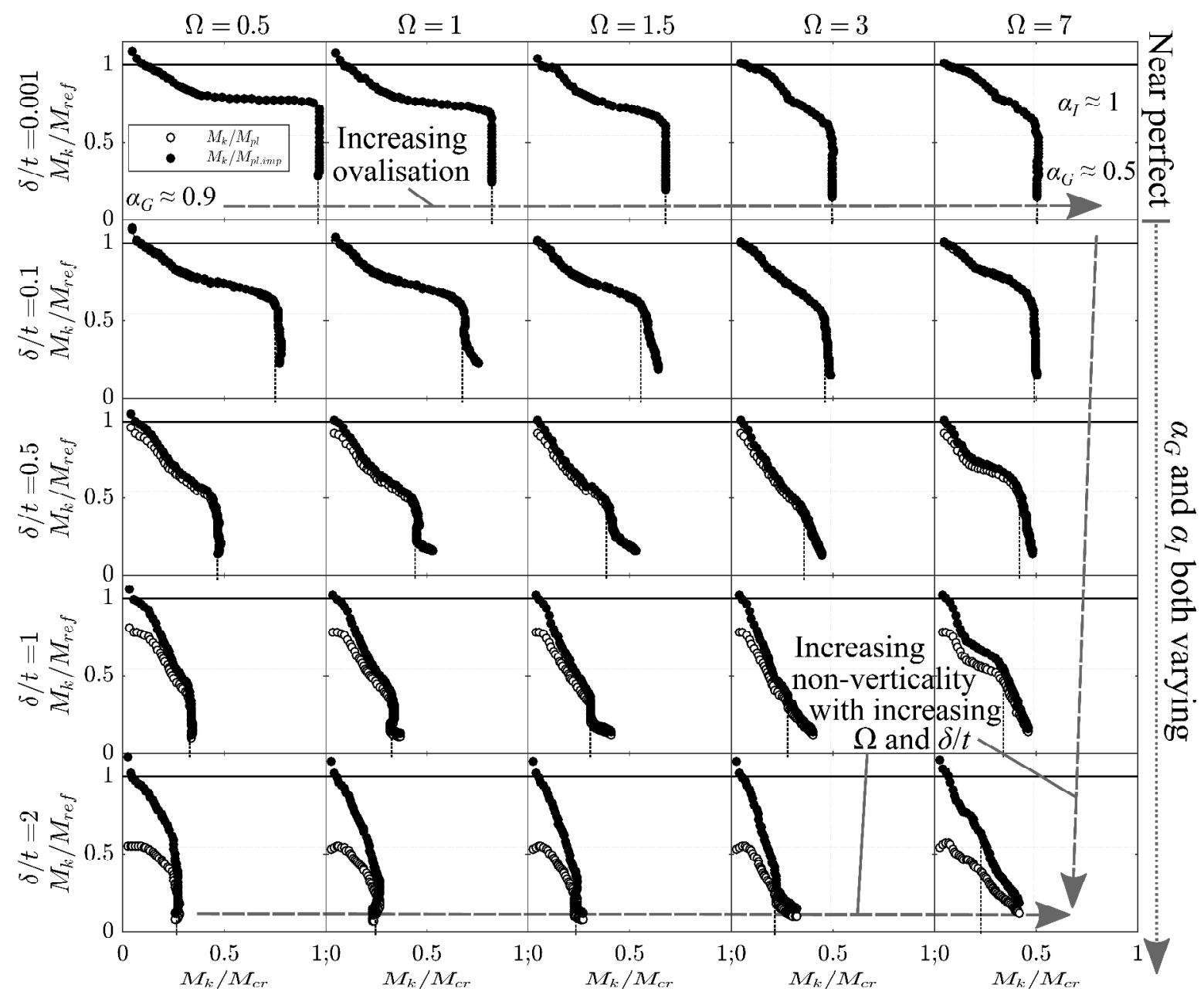

Fig. 12 - A selection of capacity curves in $M_{k} / M_{p l}$ vs $M_{k} / M_{c r}$ and $M_{k} / M_{p l, i m p}$ vs $M_{k} / M_{c r}$ spaces at constant $\delta / t$ and $\Omega$ within the 'transitional' length domain 


\section{Computed capacity curves in the 'transitional' length domain}

A selection of capacity curves established using GMNIAs for cylinders in the elastic 'transitional' length domain ( $\Omega=0.5$ to 7$)$ are presented in two alternative forms in Fig. 12 in ' $M_{k} / M_{r e f}$ ' vs $M_{k} / M_{c r}$ space for compactness. The capacity curves are shown at the fixed dimensionless lengths $\Omega=0.5$ (boundary with 'medium' domain, with no ovalisation) 1, 1.5, $3 \& 7$ (boundary with asymptotic 'long' domain, with fully-developed ovalisation) and constant imperfection amplitudes $\delta / t=0.001$ (reduced from 0.01 on the basis of conclusions from Section 6 to reflect a 'near perfect' system very closely), 0.1, $0.5,1.0 \& 2.0$. The white data points represent the capacity curve resistances expressed relative to the full plastic moment of the perfect tube where $M_{r e f}=M_{p l}$, characterised in terms of the established formulation (Eq. 2). The black data points represent the same data expressed relative to the full plastic moment of the imperfect tube where $M_{\text {ref }}=M_{p l \text { imp }}$, characterised using the modified formulation (Eq. 5). It may be seen that the revised formulation consistently preserves the physical interpretation of the RRD parameters, particularly those representing the behaviour of thick imperfect cylinders $\left(\lambda_{0, i}, \eta_{0, i}\right.$ and $\left.\chi_{h, i}\right)$. It should also be noted that cylinders that are so thick that they should approach the full plastic moment do not have imperfection amplitudes that prevent them from doing so. The parts of the curves in Fig. 12 for low slenderness shells with imperfection amplitudes in the range $0.4<\delta / t<2.0$ therefore do not represent practical structures: these parts of the curves are needed only to identify the patterns of behaviour in more slender shells in which yielding affects the strength.

The elastic region on the right-hand side of each curve for 'near perfect' cylinders is manifest as a vertical line, minor variations due to numerical issues aside, which shows that the dimensionless parameter $\Omega$ is the appropriate grouping to ensure constant geometric nonlinearity even at fully-developed ovalisation. This vertical line migrates from a position of $M_{k} / M_{c r} \approx 0.9$ at $\Omega=0.5$ to $M_{k} / M_{c r} \sim 0.5$ at $\Omega=7$ in close agreement with previous findings for $\alpha_{G}$ (Fig. 2; [21]), that display an almost 50\% reduction in the nonlinear elastic buckling resistance due to pre-buckling ovalisation. However, for $\Omega>0.5$ and $\delta / t \gg>0$ the elastic region becomes increasingly curved and increasingly illrepresented by a single value of $\alpha_{I}$ (Fig. 1). The elastic regions of capacity curves of very imperfect ovalizing cylinders grouped according to constant values of $\Omega$ are no longer invariant with slenderness, which shows that $\Omega$ no longer preserves invariant geometric 
nonlinearity under these conditions. This phenomenon was explored in more detail through a dedicated set of GNIAs by Fajuyitan and Sadowski [34] who suggested that the increasingly deep weld depression imperfection acted as a stiffening corrugation against the circumferential bending associated with ovalisation at these lengths. Fajuyitan [51] also reported that it did not appear to be possible to find an alternative dimensionless length parameter that maintained verticality of the elastic portion of the capacity curve for ovalising imperfect cylinders, despite numerous possibilities being explored. A conservative work-around that preserves the characterisation of such capacity curves within the RRD framework is presented next, together with a further exploration of the physical interpretation of the computed data.

\section{RRD parameters for cylinders under uniform bending}

\subsection{Extraction procedure}

An automatable computational methodology to extract RRD parameters from a capacity curve stored in computer memory is presented in this section. The curves are presented in terms of $M_{k} / M_{p l, i m p}$ to avoid the problem of loss of physical interpretation of the parameters shown in Fig. 5a. The procedure is illustrated schematically in Fig. 13 on a selection of computed capacity curves in both 'modified' and 'traditional' formats.

The 'elastic reduction' and 'plastic range' factors $\alpha=\alpha_{G} \alpha_{I}$ and $\beta_{i}$ respectively are best extracted from the 'modified' capacity curve (Figs $13 \mathrm{a}$ and $\mathrm{b}$ ). The value $\beta_{i}$ identifies the slenderness where plasticity first begins to significantly affect the nonlinear resistance $M_{k}$, and for near perfect or mildly imperfect systems (Fig. 13a) this is usually manifest as a clear change in behaviour with decreasing slenderness and is relatively easy to identify by visual inspection. However, in more imperfect cylinders, and in particular those with significant pre-buckling ovalisation leading to significant loss of verticality in the 'elastic' portion of the capacity curve, this point becomes increasingly ill-defined and is difficult to establish unambiguously on the basis of GMNIA data alone (Fig. 13b). For this reason, a complementary set of elastic GNIAs was performed at the same pairs of $\Omega-\delta / t$ values and the resistances compared with the GMNIAs. The point where the GNIA and GMNIA $M_{k}$ load factors begin to diverge beyond a specified tolerance (say, $10^{-3}$ ) is easily identified as a point $P$ (Figs $13 \mathrm{a}$ and b) in $M_{k} / M_{p l, i m p}$ vs $M_{k} / M_{c r}$ space whose vertical coordinate is (1 - 
$\left.\beta_{i}\right)$. For 'modified' capacity curves established at constant geometric nonlinearity, $\alpha$ should identify a vertical line on the elastic portion to the right of the horizontal axis. This was found to be largely the case in the 'medium' length domain for reasons given earlier (Fig. 11). However, for long cylinders in the 'transitional' length domain, the verticality of the curves deteriorates with growing pre-buckling ovalisation and imperfection amplitude (Fig. 12). It is proposed here to adopt the resistance coordinate of the same point $P$ above to select a value for $\alpha=\alpha_{I} \alpha_{G}$. This guarantees a conservative assessment of the imperfection sensitivity $\alpha$ for any curve, eliminating the need to consider the loss of verticality of the elastic region. The combination of $\alpha$ and $\beta_{i}$ then lead to the 'plastic limit' slenderness $\lambda_{p, i}=$ $\sqrt{ }\left(\alpha_{G} \alpha_{I} /\left(1-\beta_{i}\right)\right)$.
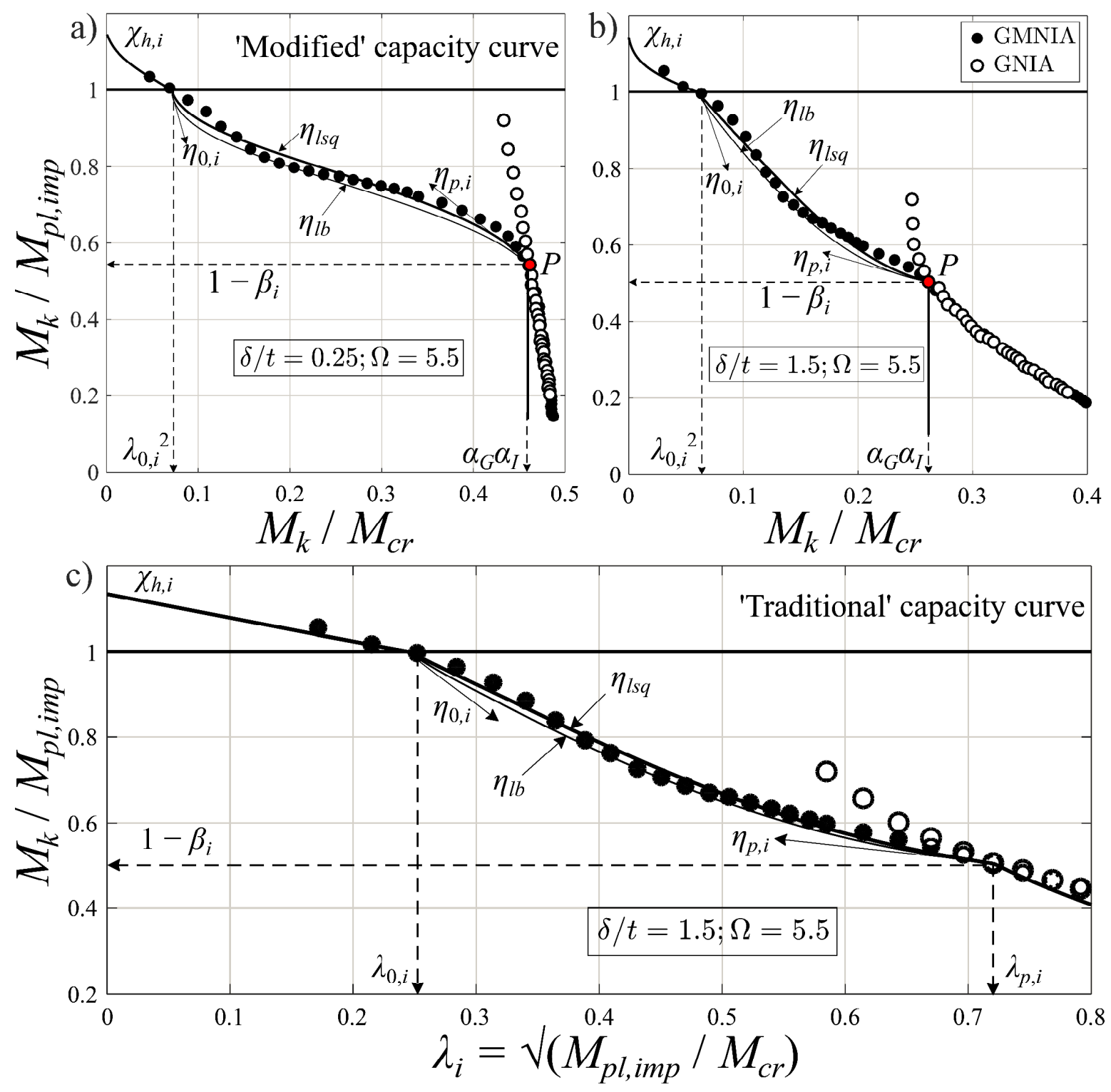

Fig. 13 - Extraction of RRD algebraic parameters from individual capacity curves. 
The remaining RRD parameters $\lambda_{0, i}, \eta_{0, i}, \eta_{p, i}$ and $\chi_{h, i}$ are best extracted from the 'traditional' form of the capacity curve (Fig. 13c). The 'squash limit' $\lambda_{0 . i}$ identifies the attainment of the full plastic condition $M_{p l, i m p}\left(M_{k} / M_{p l, i m p}=1\right)$. In general, no computed data point will be found precisely at this value, but it is sufficient to find an accurate value by linear interpolation between the two data points whose resistances are closest to unity. Where no computed point exceeded $M_{k} / M_{p l, i m p}=1$, the two data points with the lowest slendernesses were used with a backwards linear projection to obtain an approximate value for $\lambda_{0, i}$ (naturally, this could indicate the need for further calculations). The high resolution of computed data points ensured that the squash limit slenderness $\lambda_{0, i}$ was accurately identified. The resistance axis intercept of the line passing through these identified points was also used to obtain a conservative estimate of the hardening parameter $\chi_{h, i}$. Once the slendernesses $\lambda_{0, i}$ and $\lambda_{p, i}$ had been obtained, the data points corresponding to the intermediate elastic-plastic region were isolated and the interaction exponent $\eta_{i}\left(\lambda_{i}\right)$ established independently of the remaining parts of the capacity curve. Although any functional form for $\eta_{i}\left(\lambda_{i}\right)$ could be used, the linear variation proposed by Doerich and Rotter [16] between the boundary values of $\eta_{0, i}$ at $\lambda_{0, i}$ and $\eta_{p, i}$ at $\lambda_{p, i}$ and adopted into EN 1993-1-6:A1 [17] appears to be sufficiently accurate for practical purposes. The parameters $\eta_{0, i}$ and $\eta_{p, i}$ can be determined by performing least-squares fits to the GMNIA data ( $\eta_{l s q}$ in Fig. 13) as suggested in Chen et al. [45]. However, this does not always lead to a conservative algebraic characterisation of all resistances in the elastic-plastic region. An alternative approach, adopted here, is to find values of $\eta_{0, i}$ and $\eta_{p, i}$ that ensure that the characterised curve is a conservative lower bound to all computed elastic-plastic resistances in the range $\lambda_{0, i} \leq \lambda_{i} \leq \lambda_{p, i}\left(\eta_{l b}\right.$ in Fig. 13). It should be added that the proposal of Doerich and Rotter [16] to assume a value of $\eta_{p, i}$ derived directly from $\alpha_{G}, \alpha_{I}$ and $\beta_{i}$ to ensure slope continuity at $\lambda_{p, i}$ in $M_{k} / M_{p l, i m p}$ vs. $\lambda_{i}$ space (Fig. 13) was not adopted here. The discontinuity of slope at this location due to a strong interaction of the localised buckling mode with plasticity (Fig. 10) would have made this assumption unconservative for this structural system. 


\subsection{Conservative algebraic characterisation}

RRD parameters $\alpha_{G}, \alpha_{I}, 1-\beta_{i}, \lambda_{0, i}, \chi_{h, i}, \eta_{0, i}$ and $\eta_{p, i}$ extracted in the manner detailed above are presented here for different dimensionless lengths $\Omega$ and imperfection amplitudes $\delta / t$. Capacity curves in the 'medium' length domain are almost invariant with length when the boundary effects are negligible (Figs 9 and 11), and the capacity curve of the longest length in the 'medium' domain $(\omega=350)$ is the same as that for the shortest length in the 'transitional' domain $(\Omega=0.5)$. For the purposes of simplicity, conservatism and data reduction, the capacity curve at $\Omega=0.5$ is taken to apply to all shorter cylinders. The RRD parameters presented in what follows should be used in the modified capacity curve formulation given by Eq. 5 .

Although Rotter et al. [21] were the first to suggest an algebraic characterisation for $\alpha_{G}$, this paper offers a more compact relationship focusing solely on the transitional domain:

$$
\alpha_{G}=\left\{\begin{array}{cc}
0.9 & \Omega \leq 0.5 \\
0.5+(0.38 \sin (0.85 \Omega)+0.48 \cos (0.85 \Omega)) e^{-0.8 \Omega} & \Omega>0.5
\end{array}\right.
$$

This equation provides a small $10 \%$ reduction of the reference elastic critical buckling resistance $M_{c r}$ due to geometric nonlinearity in medium-length cylinders that do not ovalise at all (valid for both rotationally claimed and unrestrained end boundary conditions; Fig. 2), and reaches a 50\% reduction for fully-developed ovalisation in asymptotically long cylinders. Similarly, Fajuyitan and Sadowski [34] were the first to offer an algebraic characterisation for $\alpha_{I}$ that captured the length-dependent imperfection sensitivity of ovalising cylinders, which is reproduced in modified form here:

$$
\alpha_{I}=\frac{1}{1+\left(0.70+\frac{1.05}{1+0.42 \Omega^{2.8}}\right)(\delta / t)^{0.7}}
$$

This equation predicts the most severe imperfection sensitivity at $\Omega=0.5$ and transitions smoothly to a less severe invariant sensitivity for asymptotically long cylinders (Fig. 14).

The slenderness $\lambda_{p, i}=\sqrt{ }\left(\alpha_{G} \alpha_{I} /\left(1-\beta_{i}\right)\right)$ corresponds to the attainment of the moment $M_{k} / M_{p l, i m p}=1-\beta_{i}$ where plasticity first begins to significantly influence the bending resistance. For perfect non-ovalising thin cylinders, the limiting value of $\left(1-\beta_{i}\right)$ is the inverse of the shape factor for thin-walled circular hollow sections $(\pi / 4 \approx 0.785)$ (Fig. 10). 
However, the onset of plasticity occurs at lower moments with increasing imperfection amplitudes in medium length cylinders (i.e. at $\Omega=0.5$ ) due to earlier surface yielding at the midspan weld depression imperfection (first found by Chen et al. [45]), and for longer near-perfect cylinders (i.e. at $\delta / t \approx 0$ but with growing $\Omega$ ) due to a reduction in the elastic section modulus caused by pre-buckling ovalisation. As noted earlier, the presence of a full circumference weld depression effectively stiffens the midspan cross-section of a long cylinder against ovalisation and thus increases its moment capacity, manifest as an increase in $\left(1-\beta_{i}\right)$ at higher values of both $\Omega$ and $\delta / t$. Because this is an artificial consequence of the assumed modelled imperfection (see also Sadowski and Rotter [18]), its effect was omitted from the characterised algebraic relationship. The proposed equation for $\left(1-\beta_{i}\right)$ in Eq. 13 is compared with the computed values in Fig. 14:

$1-\beta_{i}=\frac{\pi}{4}\left(\frac{1}{1+1.3 \sqrt{\delta / t}}\right) f_{\Omega}$ where $f_{\Omega}=\left\{\begin{array}{cc}1 & \Omega \leq 0.5 \\ 0.70+\frac{0.44}{1+1.66 \Omega^{1.87}} & \Omega>0.5\end{array}\right.$

The squash limit relative slendernesses $\lambda_{0, i}$ is found to be approximately 0.3 for nearperfect non-ovalising 'medium'-length cylinders, a value that is consistent with the boundary between Class 2 and 3 circular hollow sections under bending in EN 1993-1-1 [52] at $d / t=70 \cdot \sqrt{ }\left(235 / \sigma_{y}\right)$. It also matches experimental results relatively well $[4,46,47]$. It is stressed that $\lambda_{0, i} \approx 0.3$ was obtained here assuming a linear strain hardening ratio $E_{h} / E$ of only $0.1 \%$, a conservative assumption corresponding to a statistically-significant finite positive gradient of the yield plateau in mild carbon steels [26], and is a theoretical lower bound on the possible squash limit $\lambda_{0}$ for near-perfect cylinders of 'medium' length. The effect of both imperfections and ovalisation individually is to reduce the stiffness of the fundamental equilibrium path and thus also the squash limit slenderness. However, the artificial stiffening caused by a combination of the two effects leads instead to an increase in $\lambda_{0, i}$ (Fig. 14). A conservative equation for $\lambda_{0, i}$ which ignores this stiffening effect is as follows:

$\lambda_{0, i}=\left(\frac{0.3}{1+0.4 \sqrt{\delta / t}}\right) f_{\Omega}$ where $f_{\Omega}$ is given by Eq. 13

The computed surface of extracted lower-bound estimates of the hardening limit $\chi_{h, i}$, obtained by projection of the data as described above is also shown in Fig. 14. These 
values vary between a minimum around 1.05 and a maximum around 1.4, with little discernible pattern. As this region of behaviour corresponds to the bending of very thick tubes $(r / t<\sim 20)$, a lower-bound value of $\chi_{h, i}=1.05$ is proposed for all geometries for simplicity.
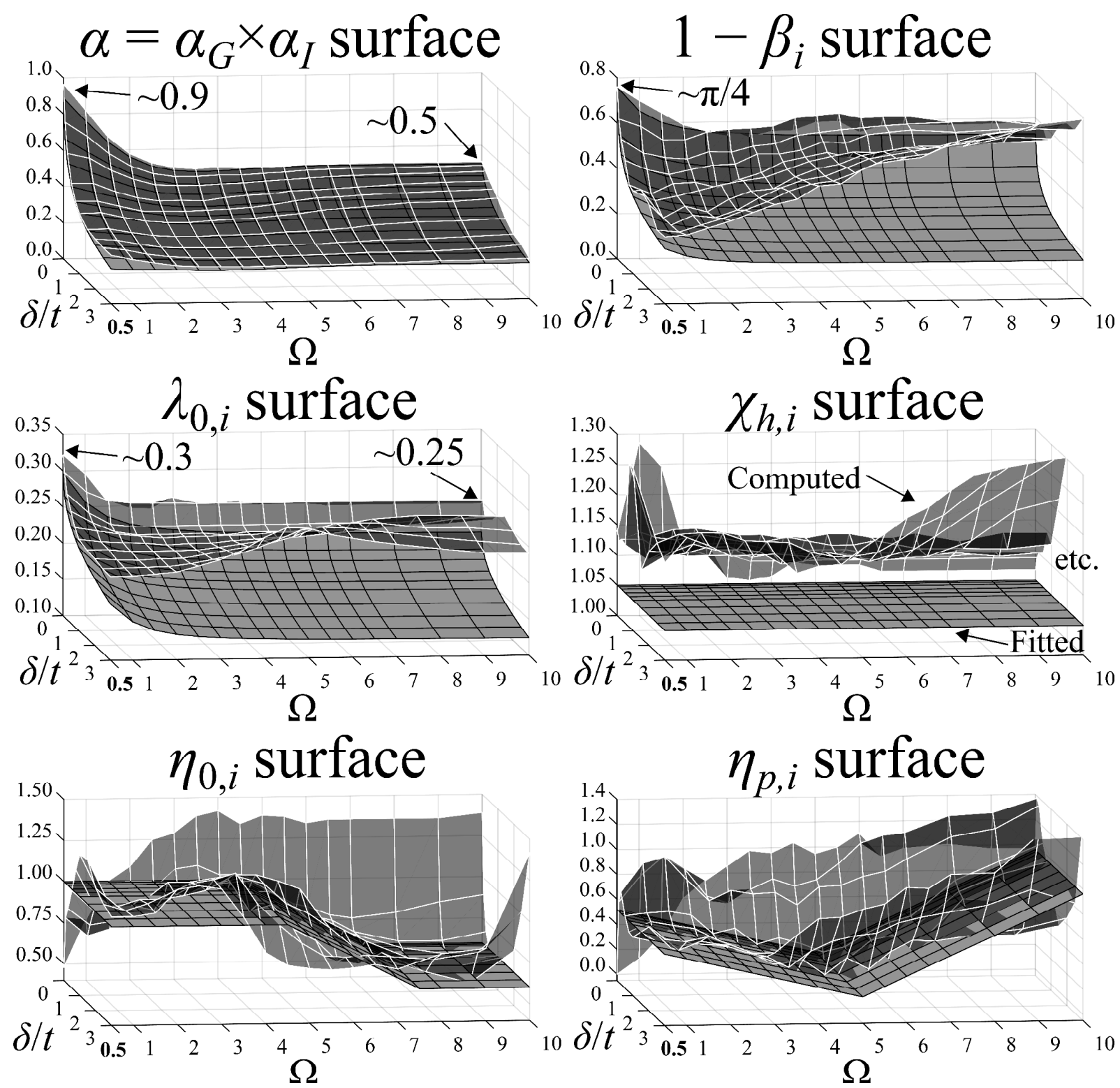

Fig. $14-3 \mathrm{D}$ surfaces of the computed and fitted $\alpha=\alpha_{G} \times \alpha_{I}, 1-\beta_{i}, \lambda_{0, i}, \chi_{h, i}, \eta_{0, i}$ and $\eta_{p, i}$ in the 'transitional' and 'long' length domains. The reader is invited to find Matlab .fig files containing the above data in the authors' ResearchGate profiles. 
Lastly, it was shown in Fig. 10 that the capacity curves of perfect non-ovalising cylinders exhibit a very flat 'yield plateau' where their bending resistance is at approximately the first yield moment $M_{e l}$ over a wide range of slendernesses. The shape of this curve is characterised by an abrupt change of slope near $M_{e l}$ and thus a near-zero value of $\eta_{p, i}$, as well as a low value of $\eta_{0, i} \approx 0.5$ corresponding to a steep descent away from $M_{p l}$, values which would make for an extremely conservative resistance characterisation if adopted universally. However, the effect of both imperfections and ovalisation is to produce more rounded and smoother curves (Figs 11 and 12) that are characterised by higher values of both $\eta_{0, i}$ and $\eta_{p, i}$ (Fig. 14). It was thus decided to base the algebraic characterisations of $\eta_{0, i}$ and $\eta_{p, i}$ on representatively imperfect cylinders, namely those which exhibit imperfection amplitudes $\delta / t$ corresponding to an average of Fabrication Tolerance Quality Classes A, B and C from EN 1993-1-6 [17,19] assuming likely $r / t$ in the range from 100 to 1000 . The proposed expressions for $\eta_{0, i}$ and $\eta_{p, i}$ are presented in Eqs 15 and 16 respectively and are visualised in Fig. 14. These are not lower bound proposals unlike those for $\alpha, \beta_{i}, \lambda_{0, i}$ and $\chi_{h, i}$ (Eqs 11 to 14), but the underlying values of $\eta_{0, i}$ and $\eta_{p, i}$ are already representative of a lower-bound fit to the GMNIA data (Fig. 13c), so the procedure does not lead to an unconservative characterisation viz a viz the simulations.

$$
\begin{gathered}
\eta_{0, i}=\left\{\begin{array}{cc}
1 & \Omega<4.5 \\
2(12-\Omega) / 15 & 4.5 \leq \Omega \leq 7.5 \\
0.6 & \Omega>7.5
\end{array}\right. \\
\eta_{p, i}= \begin{cases}0.08(7-\Omega) & \Omega<5 \\
0.16(\Omega-4) & \Omega \geq 5\end{cases}
\end{gathered}
$$

\subsection{Reliability analysis based on EN 1990 (2002 version) Annex D}

A reliability analysis was carried out based on the provisions for 'design assisted by testing' in Annex D of EN 1990 [53] to estimate values for the partial factor on the bending resistance $\gamma_{M}$ [54], defined as $\gamma_{M}=R_{n} / R_{d}$ where $R_{n}$ and $R_{d}$ are the nominal and design bending resistances respectively. A database of 141 test results for carbon steel tubes under bending was compiled from the available literature: full details may be found in the open-access paper by Wang et al. [55]. Of these, 59 specimens were classified as 'squat' according to their dimensionless cross-sectional slenderness $d /\left(t \varepsilon^{2}\right)$ which was less than 70 (approximately the internationally-established upper slenderness limit for 'Class 1 
and 2' tubular sections [56]), where $d$ and $t$ were taken as the middle surface diameter and thickness respectively (using reported measured values where possible) and $\varepsilon^{2}=235 / \sigma_{y}$ evaluated on the basis of the reported measured values of the $0.2 \%$ proof stress. The remaining 82 specimens were classified as 'intermediate'. The values of $d /\left(t \varepsilon^{2}\right)$ for these tests did not exceed $\sim 250$, with the exception of two specimens by Stephens et al. [57] which had $d /\left(t \varepsilon^{2}\right)$ of $\sim 477$ and $\sim 578$.

A detailed description of the application of this reliability procedure may be found in Afshan et al. [58] and will not be reproduced here due to space constraints. The coefficients of variation $V_{X i}$ of each $i$-th basic input random variable $X_{i}\left(\sigma_{y}, E, v, d\right.$ and $\left.t\right)$ were sourced from the background literature and are presented in Table 3 , with the exception of the tube length $L$ (specifically, the length of the uniform moment region) which was treated as deterministic due to lack of data (any deviations would anyway be negligible compared with the total length of the tube). The RRD resistance model additionally requires the specification of a local imperfection amplitude $\delta / t$, but this information is typically also not available. Consequently, values of $\delta / t$ were assumed on the basis of a deterministic categorical Fabrication Tolerance Quality Class variable $[17,19]$ such that $\delta / t=(1 / Q) \sqrt{ }(r / t)$ and the reliability analysis was performed for each of the three possible Classes: A ('Excellent', smallest imperfections; $Q=40$ ), B ('High'; $Q=25$ ) and C ('Normal', deepest imperfections; $Q=16$ ). For each Class, the analysis was additionally performed three times: on subsets of 59 'squat' and 82 'intermediate' specimens respectively and on the full data set of 141 specimens. The coefficient of variation $V_{r t}$ accounting for the variability of the resistance function $g_{r t}(\underline{\mathrm{X}})$ due to the variability of the 5 basic random variables $X_{i}$ (i.e. Eq. 5 , with RRD parameters as defined in Section 8.2) is given in Eq. 17, where $\underline{X}$ is a vector of $X_{i}^{\prime}$ s, $\underline{X}_{m}$ is a vector of their means and $\sigma_{i}$ are their standard deviations (Table 3 ). This equation was evaluated using both the first-order approximation shown on the right (with partial derivatives of the complicated RRD resistance function evaluated using finite differences) and the full form shown on the left (using a simple Monte Carlo approach), with negligible differences found in the final values of $V_{r t}$.

$V_{r t}^{2}=\frac{V A R\left[g_{r t}(\underline{\mathrm{X}})\right]}{g_{r t}^{2}\left(\underline{\mathrm{X}}_{m}\right)} \approx \frac{1}{g_{r t}^{2}\left(\underline{\mathrm{X}}_{m}\right)} \cdot \sum_{i=1}^{5}\left(\frac{\partial g_{r t}}{\partial X_{i}} \sigma_{i}\right)$ 
Table 3 - Statistics of basic variables in the resistance function.

\begin{tabular}{|c|c|c|c|c|c|}
\hline Basic variable $\left(X_{i}\right)$ & Yield stress $\sigma_{y}^{\dagger}$ & $\begin{array}{c}\text { Elastic } \\
\text { modulus } E \ddagger\end{array}$ & $\begin{array}{c}\text { Poisson } \\
\text { ratio } v_{*}\end{array}$ & $\begin{array}{c}\text { Diameter } \\
d_{*}^{*}\end{array}$ & $\begin{array}{c}\text { Thickness } \\
t_{* *}^{*}\end{array}$ \\
\hline Mean $\left(X_{i, m}\right) /$ Nominal & $\begin{array}{c}1.21(\leq 235 \mathrm{MPa}) \\
1.11(\geq 355 \mathrm{MPa})\end{array}$ & 1.03 & 1 & 1 & 0.976 \\
\hline $\begin{array}{c}\text { Coefficient of Variation } \\
\left(V_{X i}\right)\end{array}$ & $\begin{array}{c}0.076(\leq 235 \mathrm{MPa}) \\
0.065(\geq 355 \mathrm{MPa})\end{array}$ & 0.045 & 0.03 & 0.01 & 0.0095 \\
\hline
\end{tabular}

Sources: $†$ Melcher et al. [60], with linear interpolation used in the range $235 \leq \sigma_{y} \leq 355$;

†Galambos [61]; *JCSS [62]; *Sadowski et al. [33]; **Schmidt and Bartlett [63].

The EN 1990 Annex D [53] procedure was used to determine a value of $\gamma_{M}, j$ for each specimen which was then combined into an overall value of $\gamma_{M}$ as follows [58], where $N$ is the number of specimens in the considered data set (i.e. 59, 82 or 141):

$$
\gamma_{M}=\frac{\sum_{j=1}^{N} R_{n, j}^{2}}{\sum_{j=1}^{N} R_{n, j} R_{d, j}}
$$

Table 4 - Summary of reliability analysis results (note that the values in parentheses represent analysis for a reduced data set with the specimens of Sherman [2] removed).

\begin{tabular}{|c|c|c|c|c|c|}
\hline $\begin{array}{c}\text { Fabrication } \\
\text { Tolerance Quality } \\
\text { Class }\end{array}$ & $\begin{array}{c}\text { Mean value } \\
\text { correction } \\
\text { factor } b\end{array}$ & \multicolumn{2}{|c|}{ Coefficients of variation } & \multirow{2}{*}{$\begin{array}{c}\text { Overall partial } \\
\text { factor on } \\
\text { resistance } \gamma_{M}\end{array}$} \\
\cline { 5 - 6 } & & $V_{\delta}$ & $V_{r t}$ & $V_{r}$ & $1.78(1.53)$ \\
\hline A ('squat') $\dagger$ & $1.05(1.03)$ & $0.18(0.12)$ & $0.07(0.07)$ & $0.19(0.14)$ & $1.61(1.45)$ \\
\hline A ('intermediate') $\ddagger$ & $1.09(1.09)$ & $0.16(0.13)$ & $0.05(0.05)$ & $0.17(0.14)$ & $1.65(1.45)$ \\
\hline A (all)* & $1.09(1.09)$ & $0.17(0.13)$ & $0.06(0.06)$ & $0.18(0.14)$ & $1.76(1.52)$ \\
\hline B ('squat') $\dagger$ & $1.05(1.04)$ & $0.18(0.12)$ & $0.07(0.07)$ & $0.19(0.14)$ & $1.55(1.40)$ \\
\hline B ('intermediate') $\ddagger$ & $1.12(1.12)$ & $0.16(0.13)$ & $0.05(0.05)$ & $0.17(0.14)$ & $1.60(1.40)$ \\
\hline B (all)* & $1.11(1.12)$ & $0.17(0.13)$ & $0.06(0.06)$ & $0.18(0.14)$ & $1.74(1.51)$ \\
\hline C ('squat') $\dagger$ & $1.07(1.05)$ & $0.18(0.13)$ & $0.07(0.07)$ & $0.19(0.14)$ & $1.49(1.34)$ \\
\hline C ('intermediate') $\ddagger$ & $1.15(1.16)$ & $0.16(0.13)$ & $0.05(0.05)$ & $0.17(0.14)$ & $1.54(1.36)$ \\
\hline C (all)* & $1.15(1.16)$ & $0.17(0.13)$ & $0.06(0.06)$ & $0.18(0.14)$ & \\
\hline
\end{tabular}

$\dagger$ using a subset of 59 (or 53 without Sherman tests) 'squat' specimens; $d /\left(t \varepsilon^{2}\right)<70$.

$\ddagger$ using a subset of 82 (or 70 without Sherman tests) 'intermediate' specimens; $d /\left(t \varepsilon^{2}\right)>70$. * using the full data set of 141 (or 123 without Sherman tests) specimens. 
A summary of the outcomes of the reliability analysis is presented in Table 4, where $V_{r}^{2}=$ $\left(V_{\delta}^{2}+1\right)\left(V_{r t}^{2}+1\right)-1$. The mean value correction factors $b$, obtained by a least squares fit in the linear space of the observed vs predicted (by the RRD resistance function using measured inputs) test resistances $R_{o b s}$ vs $R_{\text {pred }}$ are predominantly greater than unity indicating that the RRD resistance function is on average conservative in predicting the resistance of a test (Fig. 15). The coefficient of variation of the errors $V_{\delta}$ is used to quantify the variability associated with the resistance model: a higher $V_{\delta}$ indicates a larger scatter in the predictions of test results, which in turn leads to more stringent partial factor $\gamma_{M}$ recommendations to account for uncertainty introduced by the scatter. A high scatter means that a future test result's $R_{o b s} / R_{\text {pred }}$ ratio is likely to fall within a wide range of values, potentially even such that $R_{o b s} / R_{\text {pred }}<1$. Strategies to reduce scatter include improving the resistance model, though it is difficult to envisage further enhancements to the RRD formulation without adding to the already lengthy list of algebraic parameters, or sub-dividing the data into subsets which are known to share a common factor. The latter approach was adopted by dividing the data into the 'squat' and 'intermediate' domains as discussed earlier, and it was found that the overall predicted $\gamma_{M}$ is significantly different depending on whether the analysis considers the full data set of 141 specimens at once or a slenderness-dependent subset.

The predicted $\gamma_{M}$ partial factors, all above unity, are seen to decrease with increasing imperfection amplitude (lower Fabrication Tolerance Quality Class), since the resistance function on average predicts lower resistances for tubes that are a priori assumed to be more imperfect. Additionally, the RRD resistance function is on average more accurate at predicting the test resistances of 'squat' specimens as shown in (Fig. 15) where the observed to predicted capacity ratio in that slenderness region generally falls between 0.8 and 1.5 (close to 1 where predications match observations), compared to 'intermediate' specimens which lie in the observed to predicted capacity ratio range of 1.2 and 1.8. Therefore, and perhaps somewhat surprisingly, a higher $\gamma_{M}$ of $\sim 1.75$ is necessary to reduce the nominal predictions for 'squat' specimens down to a design value corresponding to the 0.1 percentile, compared to $\gamma_{M}$ of $\sim 1.6$ for 'intermediate' specimens. The values of $b$ and $V_{\delta}$ are indicative of this; 'squat' specimens' predictions exhibit a lower $b$ and higher $V_{\delta}$, whereas those for 'intermediate' specimens exhibit a higher $b$ and lower $V_{\delta}$. 


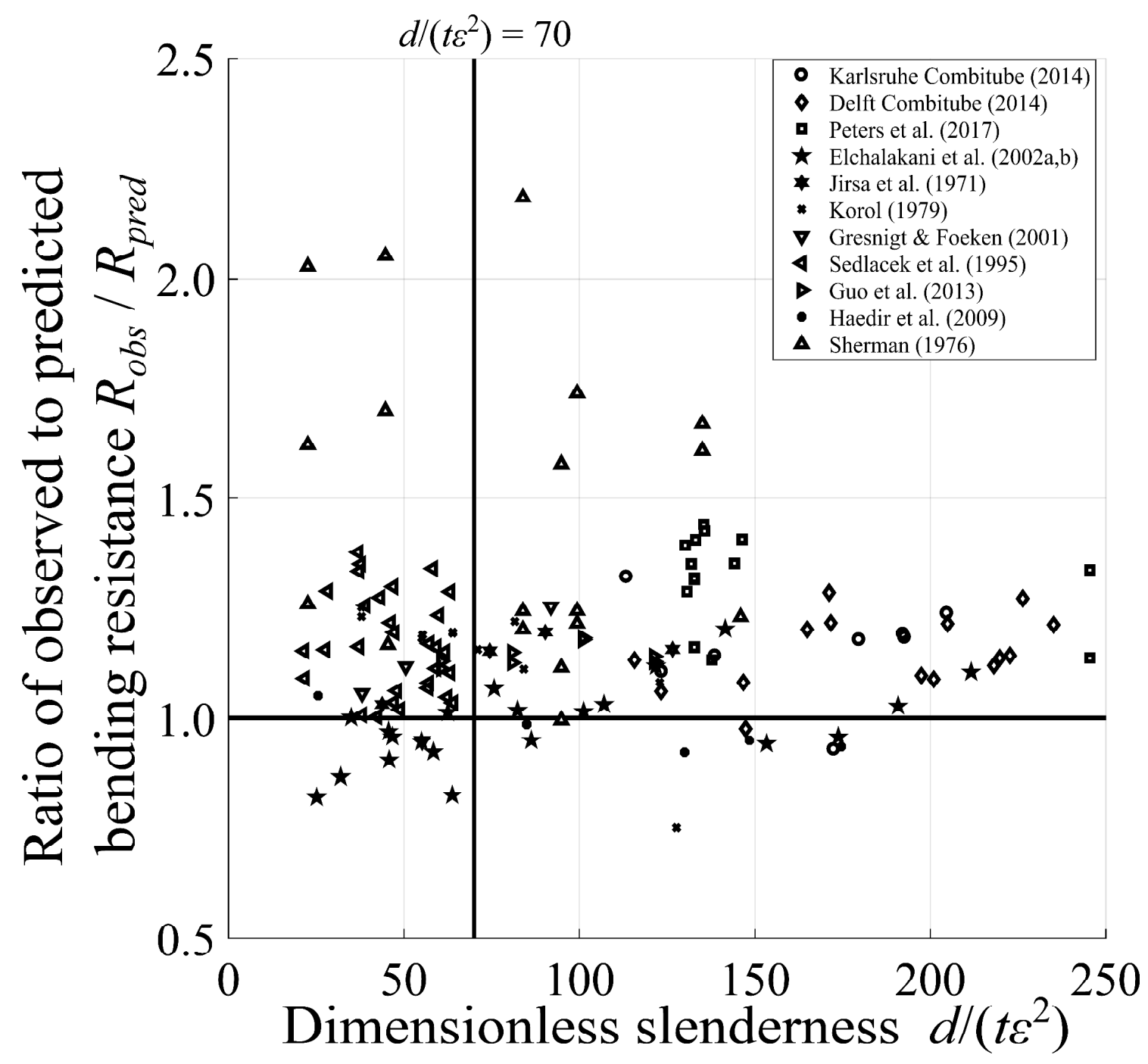

Fig. 15 - Comparison of observed to predicted bending resistances assuming Fabrication

Tolerance Quality Class C. Further references may be found in Wang et al. [55].

In considering the above analysis, it is vital for the reader to be mindful that the validity of these computed $\gamma_{M}$ partial factors is intimately dependent on the quality of the data set upon which the calibration is performed. In particular, the following are sources of "known unknowns' in the test database which contribute to the inflation of scatter and thus also the $\gamma_{M}$ factors in a way that cannot be currently quantified:

- The test database, though substantial, is comprised of specimens of mostly low slenderness that are representative of 'beam'-like CHS members that are arguably governed by EN 1993-1-1. The authors are not aware of any test programme to have systematically investigated the elastic buckling of very slender cylindrical 
shells under uniform bending other than the two specimens reported in Stephens et al. [57]. This is not surprising, given the prohibitively expensive cost of testing even a scale model of a silo or wind turbine tower to failure. This means that it is currently not possible to reliably calibrate $\gamma_{M}$ for thin shells that fall within the remit of EN 1993-1-6, and it seems unlikely to be possible for some time.

- The forms and amplitudes of the imperfections in the small-scale laboratory specimens are generally unknowable and not necessarily representative of largescale civil engineering shell structures in situ. Where such information is available (e.g. Sadowski et al. [33]), it suggests that many test specimens may be worse than Fabrication Tolerance Quality Class C of EN 1993-1-6 [17,19] and thus not necessarily representative of the quality of members in construction.

- The importance of maintaining circularity of the cross-section at the load application points in a four-point bending test arrangement has only recently been shown to be critical in preventing excessive ovalisation [59]. As many of the historic bending tests did not control for this effect, the specimens' bending resistances may be much lower than those of constructed shells and this effect may contribute towards the high experimental scatter observed.

- The database spans a timescale of over four decades and includes contributions from researchers working in different environments all over the world. Each team would have used different research methodologies and test procedures, as well as steel quality representative of the era. The very high moments apparently achieved in some of the tests by Sherman [2], for example, which contribute greatly to scatter inflation appear to be due to catenary action developed by the tested specimens being rotationally fixed at the end supports. The numbers in parentheses included in Table 4 indicate the results of the reliability analysis with the Sherman tests removed: the significantly reduced experimental scatter now leads to lower $V_{\delta}$ and thus $V_{r}$ predictions, and more favourable $\gamma_{M}$ factors. Analysts should then keep in mind that poorly-documented test data from another century may not necessarily be an appropriate basis upon which to calibrate partial factors for modern design.

In conclusion, it is evident that the very high values of $\gamma_{M}$ obtained here should be treated with caution, and some consideration given to matching current design practice when adopting a suitable value for design purposes. 


\section{Summary}

This paper has presented the most comprehensive computational study known to date of the nonlinear bending resistance of imperfect cylindrical shells under uniform bending, including the full range of possible lengths and thicknesses. A conservative algebraic characterisation has been proposed based on the processing of over 28,000 nonlinear finite element calculations for the resistance relationship within the Reference Resistance Design framework, recently adopted as a formal design concept within the European Standard on metal shells EN 1993-1-6. The capacity curve upon which the framework is based was modified to permit an accurate representation of imperfect systems of low slenderness, a crucial modelling device to enable a unified global characterisation. Finally, a reliability analysis was performed according to Annex D of EN 1990 (2002) to estimate the most probable values of partial factors on the bending resistance $\gamma_{M}$, although the authors stress that any such calibration is intimately related to the quality of the underlying test database.

\section{Acknowledgements}

This work was partly funded by the UK Engineering and Physical Sciences Research Council (EPSRC) with grant contract EP/N024060/1. O. Kunle Fajuyitan's contribution was funded by the Petroleum Technology Development Fund (PTDF) of Nigeria. The authors are grateful to Dr Peter Stafford of Imperial College London for his contribution to the reliability analysis. The reader is warmly invited to consult the authors' ResearchGate profiles for additional resources related to this study.

\section{References}

[1] Brazier L.G. (1927) "On the flexure of thin cylindrical shells and other 'thin' sections" Proc. Royal. Soc. Series A London, 116, 104-114.

[2] Sherman D.R. (1976) "Tests of circular steel tubes in bending" ASCE Journal of the Structural Division, 102(ST11), 2181-2195.

[3] Korol R.M. (1979) "Critical buckling of strains of round tubes in flexure" International Journal of Mechanical Sciences, 21, 719-730. 
[4] Sedlacek G., Dahl W., Stranghöner N. \& Kalinowski B. (1998) "Investigation of the rotation behaviour of hollow section beams" Final Report, European Commission, Technical Steel Research Contract No. 7210-SA/119, Brussels.

[5] Elchalakani M., Zhao X.L. \& Grzebieta R.H. (2002a) "Plastic mechanism analysis of circular tubes under pure bending” International Journal of Mechanical Sciences, 44, 1117-1143.

[6] Elchalakani M., Zhao X.L. \& Grzebieta R.H. (2002b) "Bending tests to determine slenderness limits for cold-formed circular hollow sections" Journal of Constr. Steel Research, 58, 1407-1430.

[7] Limam A., Lee L.H., Kyriakides S. (2010) "On the collapse of dented tubes under combined bending and internal pressure" International Journal of Mechanical Sciences, 55, 1-12.

[8] van Es S.H.J., Gresnigt A.M., Vasilikis D. \& Karamanos S. (2016) "Ultimate bending capacity of spiral-welded steel tubes - Part I: Experiments" Thin-Walled Structures, 102, 286-304.

[9] Rotter J.M. \& Sadowski A.J. (2013) "Critical evaluation of design procedures for CombiWalls: Tubular member design rules and standards" RFCS Combitube WP1 Task 1.1 Final Report, The University of Edinburgh.

[10] Rotter J.M. (2016a) "Advances in understanding shell buckling phenomena and their characterisation for practical design" in Recent Progress in Steel and Composite Structures, Eds. M.A. Giżejowski, A. Kozłowski, J. Marcinowski \& J. Ziółko, CRC Press, Taylor and Francis, London, pp. 2-15.

[11] Rotter J.M. (2016b) "The new method of Reference Resistance Design for shell structures", Proc. SDSS 2016, International Colloquium on Stability and Ductility of Steel Structures, Timisoara, Romania, pp. 623-630.

[12] Rotter J.M. (2017) "Challenges and their resolution in both philosophy and process to exploit advanced computation in shell structure design" Proc. $11^{\text {th }}$ Int. Conf. on Shell Structures: Theory and Applications, SSTA 2017, Gdańsk, Poland.

[13] Rotter J.M. (2005) "Stability and plasticity in structural analysis: a new conceptual framework" in Advances in Steel Structures, Eds Z.Y. Shen, G.Q. Li \& S.L. Chan, Elsevier, pp. 1815-1826.

[14] Rotter J.M. (2007) "A framework for exploiting different computational assessments in structural design" Proc. $6^{\text {th }}$ Int. Conf. on Steel and Aluminium Structures ICSAS 07, Oxford, UK.

[15] Rotter J.M. (2011) "Shell buckling design and assessment and the LBA-MNA methodology" Stahlbau, 80(11), 791-803.

[16] Doerich C. \& Rotter J.M. (2011) "Generalised capacity curves for stability and plasticity: Application and limitations" Thin-Walled Structures, 49, 1132-1140.

[17] EN 1993-1-6:A1 (2017) "Eurocode 3: Design of steel structures. Part 1-6: Strength and stability of shell structures. Amendment A1" Comité Européen de Normalisation, CEN, Brussels. 
[18] Sadowski A.J. \& Rotter J.M. (2011) "Steel silos with different aspect ratios: II - behaviour under eccentric discharge" Journal of Constructional Steel Research, 67, 1545-1553.

[19] EN 1993-1-6 (2007) "Eurocode 3: Design of steel structures. Part 1-6: Strength and stability of shell structures" Comité Européen de Normalisation, CEN, Brussels.

[20] Sadowski A.J., Fajuyitan O.K. \& Wang J. (2017a) "A computational strategy to establish algebraic parameters for the Reference Resistance Design of metal shell structures" Advances in Engineering Software, 109, 15-30.

[21] Rotter J.M., Sadowski A.J. \& Chen L. (2014) "Nonlinear stability of thin elastic cylinders of different length under global bending" Int. Journal of Solids and Structures, 51, 2826-2839.

[22] Yamaki N. (1984) "Elastic stability of circular cylindrical shells" Elsevier Science, NorthHolland.

[23] Fajuyitan O.K., Sadowski A.J. \& Rotter J.M. (2015) "A study of imperfect cylindrical steel tubes under global bending and varying support conditions" Proc. $8^{\text {th }}$ Int. Conf. on Advances in Steel Structures, 22-24 July, Lisbon, Portugal.

[24] Fajuyitan O.K., Sadowski A.J. \& Wadee M.A. (2017) "Buckling of very short elastic cylinders with weld imperfections under uniform bending" Proc. Eurosteel 2017 Conference, 13-15 September, Copenhagen, Denmark.

[25] Fajuyitan O.K., Sadowski A.J., Wadee M.A. \& Rotter J.M. (2018) "Nonlinear behaviour of short elastic cylindrical shells under global bending" Thin-walled Structures, 124, 574-587.

[26] Sadowski A.J., Rotter J.M., Stafford P.J., Reinke T. \& Ummenhofer T. (2017b) "On the gradient of the yield plateau in structural carbon steels" Jrnl. of Constr. Steel Rsrch., 130, 120-130.

[27] Rotter J.M. \& Teng J.G. (1989) "Elastic stability of cylindrical shells with weld depression" ASCE Journal of Structural Engineering, 115(5), 1244-1263.

[28] Rotter J.M. (1996) "Elastic plastic buckling and collapse in internally pressurised axially compressed silo cylinders with measured axisymmetric imperfections: interactions between imperfections, residual stresses and collapse", Proc. International Workshop on Imperfections in Metal Silos: Measurement, Characterisation and Strength Analysis, CA-Silo, Lyon, 119-140.

[29] Rotter J.M. (1998a) "Shell structures: the new European standard and current research needs" Thin-Walled Structures, 31, 3-23.

[30] Berry P.A., Rotter J.M. \& Bridge R.Q. (2000) "Compression tests on cylinders wit axisymmetric weld depressions" ASCE Journal of Engineering Mechanics, 126(4), 405-413.

[31] Pircher M., Berry P.A., Ding X. \& Bridge R.Q. (2001) "The shape of circumferential weldinduced imperfections in thin-walled steel silos and tanks" Thin-Walled Structures, 39, 999-1014. [32] Teng J.G., Lin X., Rotter J.M. \& Ding X.L. (2005) “Analysis of geometric imperfections in full-scale welded steel silos" Engineering Structures, 27, 938-950. 
[33] Sadowski A.J., van Es S.H.J., Reinke T., Rotter J.M., Gresnigt A.M. \& Ummenhofer T. (2015) "Harmonic analysis of initial geometric imperfections in spiral welded tubes" Engineering Structures, 85, 234-248.

[34] Fajuyitan O.K. \& Sadowski A.J. (2018) "Imperfection sensitivity in cylindrical shells under uniform bending" Advances in Structural Engineering, 21(16), 2433-2453.

[35] Sadowski A.J. \& Rotter J.M. (2013) "Solid or shell finite elements to model thick cylindrical tubes and shells under global bending" International Journal of Mechanical Sciences, 74, 143-153. [36] Xu Z., Gardner L. \& Sadowski A.J. (2017) "Nonlinear stability of elastic elliptical cylindrical shells under uniform bending" International Journal of Mechanical Sciences, 128-129, 593-606.

[37] ABAQUS (2017) “ABAQUS v. 2017" Commercial FE Software and Documentation, Dassault Systèmes, Simulia Corporation, Providence, RI, USA.

[38] Kyriakides S. \& Ju G.T. (1992a) "Bifurcation and localisation instabilities in cylindrical shells under bending - I. Experiments" International Journal of Solids and Structures, 29(9), 1117-1142. [39] Ju G.T. \& Kyriakides S. (1992b) "Bifurcation and localisation instabilities in cylindrical shells under bending - II. Predictions" International Journal of Solids and Structures, 29(0), 1143-1171. [40] Vasiliks D., Karamanos S.A., van Es S.H.J. \& Gresnigt A.M. (2016) "Ultimate bending capacity of spiral-welded steel tubes - Part II: Predictions" Thin-Walled Structures, 102, 305-319. [41] Riks E. (1979) "An incremental approach to the solution of snapping and buckling problems" International Journal of Solids and Structures, 15, 529-51.

[42] Kobayashi T., Mihara Y. \& Fujii F. (2012) "Path-tracing analysis for post-buckling process of elastic cylindrical shells under axial compression” Thin-Walled Structures, 61, 180-187.

[43] Esslinger M. (1970) "Hochgeschwindigkeitsaufnahmen von Beulvorgang dunnwandiger, axialbelasteter Zylinder" Der Stahlbau, 39(3), 73-76.

[44] Rotter J.M. (2008) "The elastic-plastic imperfection sensitivity of axially compressed cylinders with weld depressions" Proc. Eurosteel 2008 Conference, Graz, Austria, 3-5 September, pp. 1497-1502.

[45] Chen L., Doerich C. \& Rotter J.M. (2008) "A study of cylindrical shells under global bending in the elastic-plastic range" Steel Construction - Design and Research, 1(1), 59-65.

[46] Schmidt H. (1989) "Dickwandige Kreiszylinderschalen aus Stahl unter Axialdruckbelastung" Stahlbau, 58, 143-148.

[47] Sedlacek G. (2010) "Consistency of the equivalent geometric imperfections used in design and the tolerances for geometric imperfections used in execution" Document CEN/TC250 CEN/TC135 - Liaison February 2010, Comité Européen de Normalisation, Brussels.

[48] Rotter J.M. (1998b) "Development of proposed European design rules for buckling of axially compressed cylinders", Advances in Structural Engineering, 1 (4), 273-286. 
[49] Rotter J.M. (2004) "Cylindrical shells under axial compression" In Buckling of Thin Metal Shells, Spon Press, London, 42-87.

[50] Schmidt H. \& Rotter J.M. (2013) "Rules for the buckling limit state assessment using global numerical analysis" Chapter 8 in Buckling of Steel Shells European Design Recommendations, J.M. Rotter \& H. Schmidt (Eds), European Convention for Constructional Steelwork (ECCS), Brussels. [51] Fajuyitan O.K. (2018) "Length effects in elastic imperfect cylindrical shells under uniform bending" PhD Thesis, Imperial College London, UK.

[52] EN 1993-1-1 (2006) "Eurocode 3: Design of steel structures. Part 1-1: General rules and rules for buildings" Comité Européen de Normalisation, CEN, Brussels.

[53] EN 1990 (2002) "Eurocode - Basis of structural design” Comité Européen de Normalisation, CEN, Brussels.

[54] Sedlacek G. \& Kraus O. (2007) "Use of safety factors for the design of steel structures according to the Eurocodes" Engineering Failure Analysis, 14, 434-441.

[55] Wang J., Sadowski A.J. \& Rotter J.M. (2018) "Influence of ovalisation on the plastic collapse of thick cylindrical tubes under uniform bending" International Journal of Pressure Vessels and Piping, 168, 94-99.

[56] Gresnigt A.M. \& Karamanos S. (2008) "Local buckling and deformation capacity of tubes in steel structures" In: J.F. Chen, J.Y. Ooi and J.G. Teng (Eds), Structures and granular solids - from scientific principles to engineering applications, CRC Press, 199-217.

[57] Stephens M.J., Kulak G.L. \& Montgomery C.J. (1982) "Local buckling of thin walled tubular steel members" SE Report N. 103, University of Alberta, USA.

[58] Afshan S., Francis P., Baddoo N.R. \& Gardner L. (2015) "Reliability analysis of structural stainless steel design provisions” Journal of Constructional Steel Research, 114, 293-304.

[59] Liu Q., Sadowski A.J. \& Rotter J.M. (2019) “Ovalization restraint in four-point bending tests of tubes" ASCE Journal of Engineering Mechanics, 145(3), 04019009-1 to -10.

[60] Melcher J., Kala Z., Holický M., Fajkus M. \& Rozlívka L. (2004) "Design characteristics of structural steels based on statistical analysis of metallurgical products" Journal of Constructional Steel Research, 60(3-5), 795-808.

[61] Galambos T.V. (1998) "Guide to stability design criteria for metal structures" $5^{\text {th }}$ Edition, Galambos T.V. (Ed.), Wiley \& Sons Inc.

[62] JCSS (2001) "Probabilistic Model Code - $12^{\text {th }}$ draft" Joint Committee on Structural Safety, JCSS-OSTL/DIA/VROU-10-11-2000. Accessed on 11/02/14, available at https://www.jcss.byg.dtu.dk/Publications/Probabilistic_Model_Code.aspx.

[63] Schmidt B.J. \& Bartlett F.M. (2002) "Review of resistance factor for steel: data collection" Canadian Journal of Civil Engineering, 29(1), 98-108. 